Teoria de bifurcação para equações diferenciais ordinárias generalizadas e aplicações às equações diferenciais ordinárias 



\title{
Teoria de bifurcação para equações diferenciais ordinárias generalizadas e aplicações às equações diferenciais ordinárias
}

\author{
Maria Carolina Stefani Mesquita Macena
}

Orientadora: Profa. Dra. Márcia Cristina Anderson Braz Federson

Dissertação apresentada ao Instituto de Ciências Matemáticas e de Computação - ICMC-USP, como parte dos requisitos para obtenção do título de Mestre em Ciências - Matemática . VERSÃO REVISADA 
Ficha catalográfica elaborada pela Biblioteca Prof. Achille Bassi e Seção Técnica de Informática, ICMC/USP, com os dados fornecidos pelo(a) autor(a)

Macena, Maria Carolina Stefani Mesquita

Teoria de bifurcação para equações diferencias ordinárias generalizadas e aplicações às equações diferenciais ordinárias / Maria Carolina Stefani Mesquita Macena; orientadora Márcia Cristina Anderson Braz Federson. -- São Carlos, 2013. $92 \mathrm{p}$.

Dissertação (Mestrado - Programa de Pós-Graduação em Matemática) -- Instituto de Ciências Matemáticas e de Computação, Universidade de São Paulo, 2013.

1. equações diferenciais ordinárias. 2. teoria de bifurcação para equações diferenciais ordinárias generalizadas. 3. integração de Kurzweil-Henstock. I. Anderson Braz Federson, Márcia Cristina, orient. II. Título. 
Aos meus pais Noemia e Alencar e aos amigos. 



\section{Agradecimentos}

Eu agradeço em primeiro lugar a Deus, o meu salvador, pois é ele quem me sustenta todos os dias da minha vida e quem me deu forças diante das dificuldades que enfrentei até o presente momento. Cheguei até aqui, não pelas minhas próprias mãos, mas sim pela sabedoria infinita e pelo espírito santo de Deus, o qual me direcionou e me fez vencer cada obstáculo, e me fez acreditar que eu tudo posso naquele que me fortale! Eu te amo Senhor!

Eu agradeço a minha mãe Noemia Stefani, a pessoa mais importante da minha vida, o meu esteio em todos os sentidos e a minha segurança. Agradeço, pois se hoje tenho gosto pelos estudos, eu devo a você mãe, por cada vez que me incentivou, e, a cada dia que eu pensei que não fosse conseguir, senti a sua mão, o seu apoio, e as suas palavras, me ensinando a lição mais importante que aprendi na vida: a nunca desistir dos meus sonhos e lutar com honestidade por eles. Eu agradeço ao meu pai Alencar Mesquita Macena, pelo dom da vida, pelo seu amor enquanto pude conviver contigo, pelo o que me ensinou e mais uma vez dedico esta vitória a você, pois tenho certeza que a sua partida não fez com que nos separássemos, de certo modo, acredito que esteja feliz com as minhas conquistas, te amo pai!

Eu agradeço muito a minha orientadora Profa Dra Márcia Cristina Anderson Braz Federson, por sua amizade, apoio e empenho na realização deste trabalho. Agradeço imensamente pela sua confiança em meu trabalho, pela oportunidade que me concedeu, a maturidade que desenvolvi ao seu lado, a responsabilidade que adquiri e sobretudo pela fato mais importante que aprendi contigo, que o infinito é o limite, e que nós podemos evoluir muito se quisermos, 
basta sermos valentes para isso. A minha evolução só foi possível nestes dois últimos anos a começar pelo meu esforço, mas sobretudo por você ter acreditado em mim e me dado esta oportunidade. Meus mais sinceros agradecimentos por tudo.

Eu agradeço ao Professor Jean Mawhin por todo a apoio e colaboração na realização deste trabalho e por ser uma pessoa tão humilde.

Eu agradeço a Jaqueline Godoy Mesquita, pela amizade, sinceridade, por ser o ser humano iluminado que é, livre de qualquer tipo de inveja e de uma bondade infinita, e por todas estas razões que chegou aonde está hoje. Eu te agradeço pela sua torcida verdadeira, e principalmente por toda a ajuda na realização deste trabalho.

Eu agradeço ao meu tio Luis Paiva, por todas as vezes que me incentivou a estudar, por desde quando eu era uma criança, ter olhado os meus cadernos e corrigido as minhas lições, um fato extremamnete pequeno, mas que me marcou pelo resto da minha vida, e também por este simples gesto, que estou aqui hoje escrevendo esta esta dissertação. Eu te agradeço pelo apoio em todos os sentidos e eu te amo muito! Agradeço a minha tia Fátima Mesquita de Paiva pelo seu amor verdadeiro e por sua torcida sincera, eu te amo!

Eu agradeço a minha amiga e irmã Mayara Bonani pela sua amizade sincera, por todas as vezes que me incentivou, me apoiou, por sempre se alegar de forma verdadeira com as minhas vitórias, mas sobretudo também, por sua ajuda e empenho a conquistá-las, e agradeço também, por sempre me relembrar do amor Deus em minha vida.

Eu agradeço muito a Daiane Alice Henrique pela amizade, compahia, força nos dias difíceis que passamos no começo do mestrado e por tudo o que me ajudou nestes dois anos. Agradeço ao meu amigo e companheiro Jean Berni por toda a amizade e todas as vezes que me ajudou, por ter me ensinado a estudar (sei que foi pela sua didática que enxerguei o caminho certo) e ter me ensinado também a lição mais importante: a nunca desistir de nada. Amigo se estou aqui hoje, falo de verdade, primeiramente foi por Deus, mas saiba que você teve muita contribuição para isso. Muito obrigada! Agradeço ao meu amigo Jorge Parejas pela amizade, compahia, por sua imensa bondade, sinceridade, sua paciência, incentivo e por toda a ajuda acadêmica que me proporcionou. 
Agradeço a Profa Dra Marta Cilene Gadotti por todo o apoio e orientação durante a graduação, por tudo o que me ensinou na área acadêmica, na vida e como ser humano. Agradeço a Deus por ter tido a oportunidade de te conhecer, pela sua amizade e por tudo o que já me ajudou e por sempre me lembrar do amor de Deus e de nossa mãe Maria.

Agradeço a Profa Dra Elíris Cristina Rizziolli pelo apoio, amizade verdadeira, por ser esta pessoa de caráter e íntegra que é, por todas as vezes que sempre me ajudou com muita boa vontade e atenção e, também, por sua torcida verdadeira.

Agradeço ao Prof Dr Vanderlei Marcos do Nascimento, pela amizade, por tudo o que me ajudou durante o período da graduação, pelo apoio nos momentos difíceis, os quais foram os que eu mais precisei e sempre pude contar contigo. Muito obrigada! 



\section{Resumo}

Neste trabalho, estudamos a teoria de bifurcação para equações diferenciais ordinárias (escrevemos simplesmente EDOs), bem como a existência de ponto de bifurcação para soluções periódicas destas equações. Em seguida, desenvolvemos a teoria, até então inexistente, sobre bifurcação para equações diferenciais ordinárias generalizadas (EDOs generalizadas). Neste desenvolvimento, obtivemos para EDOs generalizadas, um resultado sobre existência de ponto de bifurcação para soluções periódicas. Em seguida, através da correspondência entre EDOs e EDOs generalizadas, obtivemos novos resultados sobre a existência de ponto de bifurcação para soluções periódicas para EDOs clássicas, agora sob a ótica das EDOs generalizadas, quando então, em vez de funções continuamente diferenciáveis, necessitamos, apenas, que as funções envolvidas na EDO sejam integráveis no sentido de Kurzweil-Henstock. Adicionamos, também, um resultado sobre a existência de soluções pediódicas de EDOs generalizadas e aplicamos tal resultado para EDOs. A fim de obtermos os resultados que pretendíamos, utilizamos a teoria do grau coincidente. Finalmente, mencionamos que os resultados inéditos deste trabalho estão contidos em [6]. 



\section{Abstract}

In this work, we study the bifurcation theory for ordinary differential equations (we write simply ODEs), as well as the existence of a bifurcation point for periodic solutions of these equations. Then we develop the theory of bifurcation for generalized ordinary differential equations (we write generalized ODEs for short). Such theory is new. We obtained an existence result of a bifurcation point for periodic solutions of generalized ODEs. By means of the correspondence of classic ODEs and generalized ODEs, we were able to translate the results to classic ODEs, now in the framework of generalized ODE. This means that instead of the classic hypothesis that the functions involved in the differential equation are continuously differentiable, we only require that they are Kurzweil-Henstock integrable. We also added a result on the existence of a periodic solution of a generalized ODE and we applied such result to classic ODEs. In order to obtain our main results, we employed the coincidence degree theory. Finally, we point out that our results are contained in [6]. 



\section{Sumário}

Introdução

1 Preliminares $\quad 17$

1.1 Equações diferenciais lineares autônomas . . . . . . . . . . . . . . . . . . 17

1.2 Sistemas lineares autônomos e plano de fase . . . . . . . . . . . . . . . . 20

2 Teoria de bifurcação para EDOs 31

3 Teoria de bifurcação para EDOs generalizadas $\quad 51$

3.1 Fundamentos da teoria de EDOGs . . . . . . . . . . . . . . . . 52

3.2 Soluções periódicas de EDOGs . . . . . . . . . . . . . . . . . . 56

3.3 Existência de soluções periódicas de EDOGs . . . . . . . . . . . . . . 61

3.4 Teoria de bifurcação para EDOGs . . . . . . . . . . . . . . 66

3.5 Aplicações . . . . . . . . . . . . . . . . . . . . . . 77

$\begin{array}{ll}\text { Apêndice: } & 83\end{array}$

$\begin{array}{ll}\text { A A Integral de Kurzweil-Henstock } & 85\end{array}$

A.1 Calibres e partições . . . . . . . . . . . . . . . . . . . 85 
A.2 Integral de Kurzweil-Henstock . . . . . . . . . . . . . . . . . . . 86

A.3 Propriedades básicas da K-integral . . . . . . . . . . . . . . . . . . . . . . . 88 


\section{Introdução}

Nesta dissertação, o nosso objetivo é estudar a teoria de bifurcação em equações diferenciais ordinárias (EDOs) e, posteriormente, adaptar os resultados estudados às equações diferenciais ordinárias generalizadas (EDOGs), desenvolvendo a teoria, até então inexistente, sobre bifurcação para EDOGs. Como consequência, aplicaremos os resultados obtidos às EDOs clássicas, em condições mais gerais dos que aquelas encontradas na literatura, de modo que os resultados existentes sejam generalizados. Tais generalizações se dão pelo fato de considerarmos a integral definida por Jaroslav Kurzweil como pano de fundo das EDOGs.

Para atingirmos nosso objetivo, vamos, primeiramente, relembrar ao leitor os conceitos principais da teoria de EDOs, conceitos estes que utilizaremos nos Capítulos 2 e 3. Assim, o Capítulo 1 será dedicado a uma breve introdução às EDOs, relembramos conceitos básicos sobre EDOs lineares, bem como sistemas de equações diferenciais lineares de ordem $2 \times 2$. Apresentamos alguns exemplos destes sistemas, as suas soluções e o retrato de fase. As principais referências para este capítulo podem ser encontradas em [3] e [2].

No Capítulo 2, estudaremos o conceito de ponto de bifurcação de soluções periódicas de uma equação diferencial ordinária dependente de parâmetro. Através de um resultado que nos permite fazer uma correspondência entre os zeros de uma equação que envolve o operador $\Phi$ e as soluções periódicas de uma EDO, então será equivalente analisar o problema de bifurcação de uma equação que envolve este operador e o problema de bifurcação para soluções periódicas da EDO. Também, utilizando a teoria coincidente do grau de Brouwer, 
teremos um resultado que nos garante a existência de ponto de bifurcação para uma equação que envolve o operador $\Phi$ e apresentaremos exemplos que ilustram a teoria. A principal referência para este capítulo pode ser encontrada em [1].

Motivados pelas ideias presentes no Capítulo 2 sobre a teoria de bifurcação para EDOs, o Capítulo 3 é constituído pelos resultados estendidos sobre a teoria de bifurcação em EDOs às EDOGs e apresentaremos um resultado, o qual sob certas condições, existirá ponto de bifurcação de uma solução periódica de uma EDOG. Também, teremos um resultado que nos diz que, sob certas condições existe pelo menos uma solução periódica da EDOG. A integral explorada no capítulo 3 deve ser entendida no sentido de Kurzweil-Henstock e para mais detalhes veja o Apêndice. A principal referência para este capítulo é [6]. 


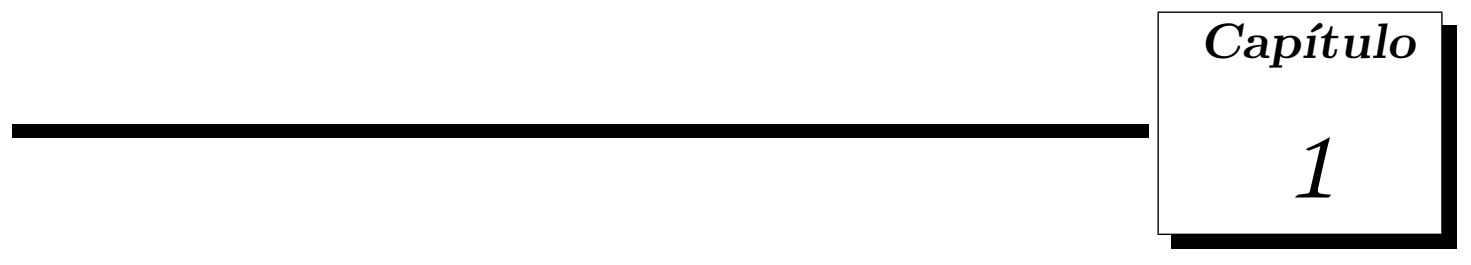

\section{Preliminares}

Neste capítulo, faremos uma revisão sobre equações diferenciais ordinárias, bem como sistemas de equações diferenciais ordinárias lineares de ordem $2 \times 2$. Apresentaremos exemplos destes sistemas, as suas soluções e os retratos de fase. As referências utilizadas para a compreensão destes fatos podem ser encontradas em [3] e [2].

\subsection{Equações diferenciais lineares autônomas}

Uma equação diferencial na qual a variável independente não aparece explicitamente no lado direito da equação é chamada autônoma. Se $t \in \mathbb{R}$ for a variável independente, uma equação diferencial autônoma de primeira ordem poderá ser escrita na forma

$$
\frac{d x}{d t}=f(x)
$$

onde $f: \mathbb{R} \rightarrow \mathbb{R}$ é uma função. 
Diremos que um número real $c$ é um ponto crítico de (1.1), se $c$ for um zero de $f$, isto é, $f(c)=0$. Se $c$ for um ponto crítico de $(1.1)$, então $x(t)=c$ será uma solução constante da equação autônoma. Uma solução constante $x(t)=c$ de (1.1) é chamada solução de equilíbrio. Também dizemos que os equilíbrios são as soluções constantes de (1.1). Podemos dizer quando uma solução não constante $x=x(t)$ de (1.1) é crescente ou decrescente, analisando o sinal da derivada $\frac{d x}{d t}$. Fazemos isso identificando os intervalos nos quais $f(x)$ é positiva ou negativa.

Exemplo 1.1. Considere a equação diferencial

$$
\frac{d P}{d t}=P(a-b P), \quad a>0, b>0
$$

a qual tem a forma

$$
\frac{d P}{d t}=f(P)
$$

onde $f(P)=P(a-b P)$. Notemos que a equação (1.2) é autônoma. Igualando a equação (1.2) a zero, vemos que 0 e $\frac{a}{b}$ são os pontos críticos da equação e, portanto, as soluções de equilíbrio são $P(t)=0$ e $P(t)=\frac{a}{b}$. Colocando os pontos críticos sobre uma reta vertical, dividimos esta reta em três intervalos: $(-\infty, 0),\left[0, \frac{a}{b}\right)$ e $\left[\frac{a}{b}, \infty\right)$.

Na Figura 1.1 a seguir, a posição das setas sobre a reta indicam o sinal de $f(P)=$ $P(a-b P)$ nesses intervalos e indicam, também, quando uma solução é crescente (seta para cima) ou decrescente (seta para baixo) em determinado intervalo.

No intervalo $(-\infty, 0)$, o sinal de $f(P)$ é negativo e, portanto, $P(t)$ decresce. Logo a seta na figura aponta para baixo. Analogamente, para o intervalo $\left(0, \frac{a}{b}\right)$ o sinal de $f(P)$ é positivo. Logo $P(t)$ é crescente e, assim, a seta na figura aponta para cima. No intervalo $\left(\frac{a}{b}, \infty\right)$, o sinal de $f(P)$ é negativo. Portanto $P(t)$ é decrescente e a seta na figura aponta para baixo.

A Figura 1.1 é chamada retrato de fase unidimensional ou simplesmente retrato de fase da equação diferencial (1.2). A reta vertical da Figura 1.1 é chamada reta de fase. 


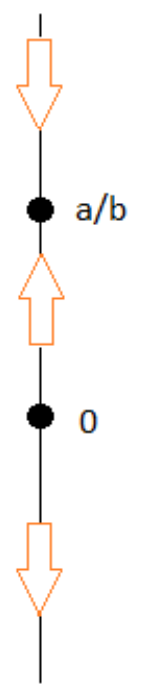

Figura 1.1: Retrato de fase da equação (1.2).

Agora, vamos estudar o comportamento das soluções de equações diferenciais ordinárias autônomas (1.1) nas proximidades de um ponto crítico $c \in \mathbb{R}$, onde $f: \mathbb{R} \rightarrow \mathbb{R}$ é uma função. Para isso, vamos analisar o retrato de fase unidimensional da equação diferencial dada em (1.1) e observar que a classificação dos pontos críticos depende da posição das flechas no retrato de fase em torno do ponto crítico.

Quando as pontas das setas em ambos os lados de $c$ apontam em direção a $c$, todas as soluções $x(t)$ de (1.1), que começam em um ponto inicial $x_{0}$ suficientemente próximas a $c$, exibem um comportamento assintótico, isto é,

$$
\lim _{t \rightarrow \infty} x(t)=c
$$

Neste caso, c será assintoticamente estável ou atrator.

Se as pontas das setas estiverem ambas opostas a $c$, então as soluções que começam perto de $c$ vão se mover, se afastando de $c$ à medida que $t$ aumentar. Neste caso, diremos que $c$ é um ponto crítico instável ou repulsor. 
Se as setas apontarem em direções opostas a $c$, então uma solução, começando em um ponto inicial $x_{0}$ suficientemente próximo a $c$, será atraída para $c$ por um lado e repelida de $c$ pelo outro. Neste caso, o ponto crítico não será atrator nem repulsor e o chamaremos ponto crítico semiestável.

\subsection{Sistemas lineares autônomos e plano de fase}

Pela dificuldade de se encontrar soluções de equações diferenciais analiticamente, é importante sabermos informações qualitativas das soluções, sem de fato resolvê-las. Para conseguir este estudo qualitativo das equações diferenciais, vamos considerar o sistema linear homogêneo de primeira ordem com coeficientes constantes da forma

$$
\left\{\begin{array}{l}
\dot{x}=a x+b y \\
\dot{y}=c x+d y
\end{array}\right.
$$

o qual pode ser escrito na forma matricial

$$
\left(\begin{array}{l}
\dot{x} \\
\dot{y}
\end{array}\right)=\left(\begin{array}{ll}
a & b \\
c & d
\end{array}\right)\left(\begin{array}{l}
x \\
y
\end{array}\right)
$$

onde $a, b, c, d \in \mathbb{R}$. O sistema (1.4) também pode ser escrito na forma vetorial

$$
\dot{X}=A X
$$

onde

$$
X=\left(\begin{array}{l}
x \\
y
\end{array}\right) \text { e } A=\left(\begin{array}{ll}
a & b \\
c & d
\end{array}\right)
$$

Utilizando o que já sabemos sobre equações diferenciais de primeira ordem, as soluções para o sistema acima terão a forma $x(t)=v e^{\lambda t}$, onde $v$ é um vetor coluna $2 \times 1, \lambda$ é um número real e $t$ é a variável independente. 
Derivando a função $x(t)$ e substituindo $x(t)$ e $\dot{x}(t)$ na equação $(1.5)$, obtemos

$$
(A-\lambda I) v=0 .
$$

Portanto $\lambda$ deve ser um autovalor da matriz $A$ e $v$ é seu autovetor associado à matriz $A$.

Os autovalores da matriz $A$ são as raízes da equação polinomial

$$
\operatorname{det}(A-\lambda I)=0
$$

isto é, são as raízes da equação

$$
\begin{gathered}
\operatorname{det}(A-\lambda I)=(a-\lambda)(d-\lambda)-b c= \\
=\lambda^{2}-(a+d) \lambda+(a d-b c)=\lambda^{2}-\operatorname{tr}(A) \lambda+\operatorname{det} A=0,
\end{gathered}
$$

onde $\operatorname{tr} A$ e $\operatorname{det} A$ denotam, respectivamente, o traço e o determinante da matriz $A$. Assim os autovalores de $A$ são dados por

$$
\lambda_{1}, \lambda_{2}=\frac{\operatorname{tr} A \pm \sqrt{(\operatorname{tr} A)^{2}-4 \operatorname{det} A}}{2} .
$$

Considere o sistema dado por $\dot{X}=A X$, como na equação (1.5). Os pontos $x \in \mathbb{R}^{2}$ para os quais $A x=0$ são chamados pontos de equilíbrio ou pontos críticos do sistema. Admitindo-se que $\operatorname{det} A \neq 0$, ou seja, que $A$ é invertível, então $X=(0,0)$ é o único ponto crítico do sistema.

Uma solução $X: \mathbb{R} \rightarrow \mathbb{R}^{2}$ do sistema linear $\dot{X}=A X$ pode ser considerada como uma representação paramétrica de uma curva no plano. Podemos olhar para essa curva como um caminho ou trajetória percorrida por uma partícula em movimento, cuja velocidade $\dot{X}$ é especificada pela equação diferencial. O plano $x y$ é chamado plano de fase e um conjunto representativo de trajetórias é chamado retrato de fase. Assim, ao analisar o sistema linear (1.5), precisamos considerar diversos casos, dependendo da natureza dos autovalores de $A$. 
Os possíveis casos para os autovalores de $A$ são analisados a seguir.

Caso 1. Os autovalores $\lambda_{1}$ e $\lambda_{2}$ de $A$ são reais e distintos, mas de mesmo sinal. Sejam $v_{1}$ e $v_{2}$ os autovetores correspondentes aos autovalores $\lambda_{1}$ e $\lambda_{2}$ de $A$. Neste caso, a solução geral da equação (1.5) é dada por

$$
\varphi(t)=c_{1} v_{1} e^{\lambda_{1} t}+c_{2} v_{2} e^{\lambda_{2} t}
$$

onde $\varphi(t)=(x(t), y(t))$. Se $\lambda_{2} \leq \lambda_{1}<0$, então $\varphi(t) \rightarrow 0$ quando $t \rightarrow \infty$, e o ponto crítico é chamado nó atrator ou nó estável. Por outro lado, se $\lambda_{2}>0$ e $\lambda_{1}>0$, então $x(t) \rightarrow \infty$ quando $t \rightarrow \infty$ e o ponto crítico é um nó repulsor ou nó instável.

Antes de seguirmos para o Caso 2, vamos dar um exemplo para ilustrar o Caso 1.

Exemplo 1.2. Considere o sistema

$$
\left(\begin{array}{l}
\dot{x} \\
\dot{y}
\end{array}\right)=\left(\begin{array}{cc}
-1 & -1 \\
0 & -\frac{1}{4}
\end{array}\right)\left(\begin{array}{l}
x \\
y
\end{array}\right)
$$

O único ponto de equilíbrio do sistema (1.6) é a origem $(0,0)$. Os autovalores da matriz característica $A$ são

$$
\lambda_{1}=-1 \text { e } \lambda_{2}=-\frac{1}{4}
$$

Os autovetores associados aos respectivos autovalores são

$$
v_{1}=(1,0) \text { e } v_{2}=(4,-3)
$$

Portanto a solução geral do sistema (1.6) é dada por

$$
\varphi(t)=c_{1}(1,0) e^{-t}+c_{2}(4,-3) e^{\frac{-t}{4}} .
$$

Vamos ilustrar a solução de (1.6) através do retrato de fase mostrado pela figura a seguir. Neste caso, o ponto crítico é um nó atrator. 


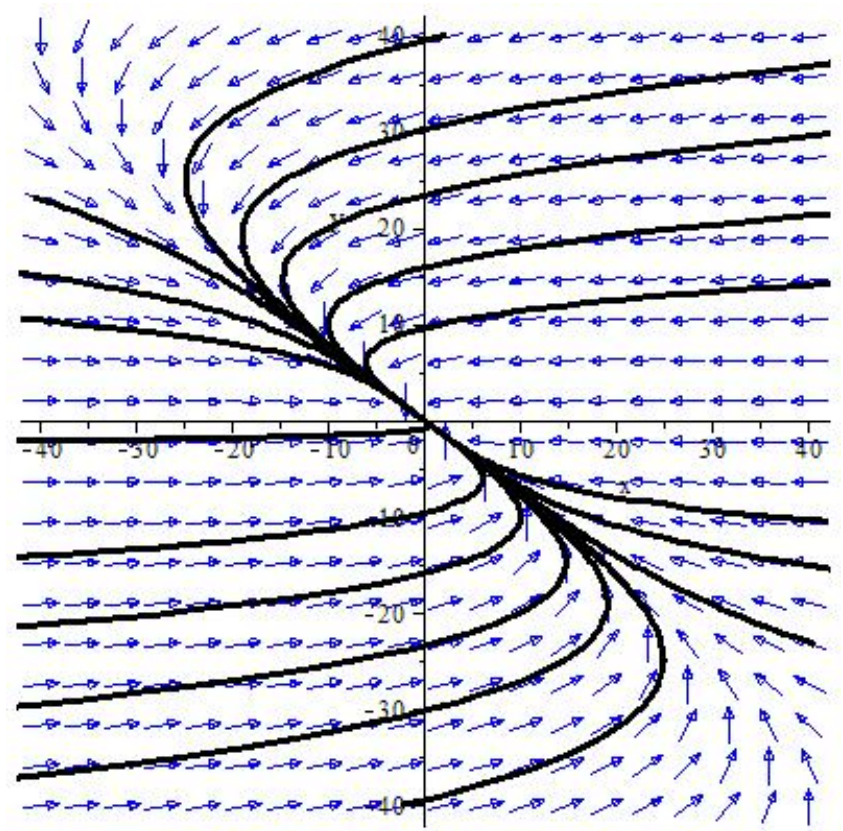

Figura 1.2: Nó atrator

Caso 2. Os autovalores $\lambda_{1}$ e $\lambda_{2}$ de $A$ são reais com sinais opostos. Neste caso, a solução geral da equação (1.5) é dada por

$$
\varphi(t)=c_{1} v_{1} e^{\lambda_{1} t}+c_{2} v_{2} e^{\lambda_{2} t}
$$

onde $x(t)=v_{1} e^{\lambda_{1} t}, y(t)=v_{2} e^{\lambda_{2} t}$ e $\lambda_{1}<0<\lambda_{2}$, e o ponto crítico é chamado ponto de sela. A solução $x(t)$ decresce exponencialmente, enquanto que a solução $y(t)$ cresce exponencialmente.

As órbitas no eixo $x$ apontam em direção à origem e as órbitas do eixo $y$ apontam para longe da origem. As órbitas fora dos eixos pertencem às hipérboles representadas na Figura 1.3 a seguir. O exemplo a seguir ilustra o Caso 2.

Exemplo 1.3. Considere o sistema

$$
\left(\begin{array}{l}
\dot{x} \\
\dot{y}
\end{array}\right)=\left(\begin{array}{cc}
-1 & 8 \\
1 & 1
\end{array}\right)\left(\begin{array}{l}
x \\
y
\end{array}\right) \text {. }
$$


O único ponto de equilíbrio de (1.7) é a origem $(0,0)$. Os autovalores da matriz característica

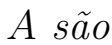

$$
\lambda_{1}=3 \text { e } \lambda_{2}=-3
$$

e os autovetores associados são dados, respectivamente, por

$$
v_{1}=(2,1) \text { e } v_{2}=(-4,1)
$$

Logo a solução geral do sistema (1.7) é da forma

$$
\varphi(t)=c_{1}(2,1) e^{3 t}+c_{2}(-4,1) e^{-3 t}
$$

O retrato de fase de (1.7) é dado pela figura a seguir.

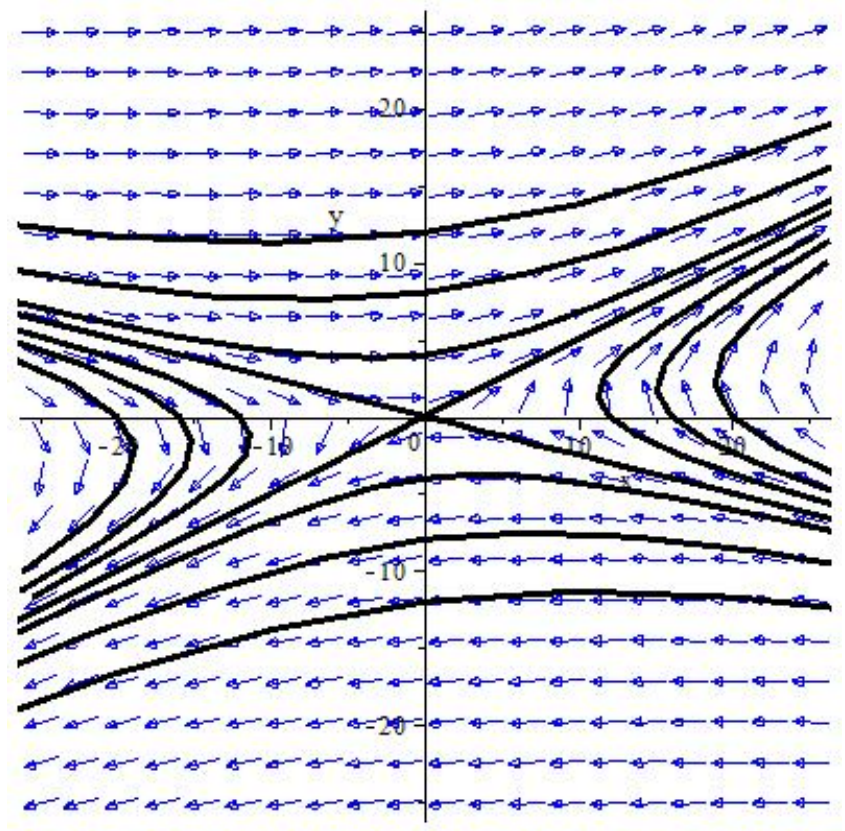

Figura 1.3: Sela

Caso 3. Os autovalores $\lambda_{1}$ e $\lambda_{2}$ de $A$ são reais e iguais. Temos dois subcasos a considerar: se o autovalor repetido tiver dois autovetores indepedentes ou somente um. 
a) Dois autovetores linearmente independentes. Seja $\lambda$ o autovalor associado aos autovetores linearmente independentes $v_{1}$ e $v_{2}$. Novamente, a solução é dada por

$$
\varphi(t)=c_{1} v_{1} e^{\lambda t}+c_{2} v_{2} e^{\lambda t}
$$

Todas as trajetórias das soluções, exceto a solução nula, estão contidas em semiretas que passam pela origem. Se $\lambda<0$, todas as soluções convergirão para a origem quando $t \rightarrow \infty$. Entretanto se $\lambda>0$, as soluções se afastarão da origem quando t crescer. Neste caso, o ponto crítico será chamado nó impróprio (atrator ou repulsor).

b) Um autovetor linearmente independente. Neste caso, a solução do sistema (1.5) é dada por

$$
\varphi(t)=c_{1} v_{1} e^{\lambda t}+c_{2}\left(v_{1} t e^{\lambda t}+v_{2} e^{\lambda t}\right)
$$

onde $v_{1}$ é um autovetor associado ao autovalor repetido $\lambda$. Para $t$ suficientemente grande, o termo dominante nesta equação é $c_{2} v_{1} t e^{\lambda t}$. Logo, se $\lambda<0$, quando $t \rightarrow \infty$, todas as trajetórias de $\varphi(t)$ tendem à origem e são tangentes à reta na direção do autovetor $v_{1}$. Analogamente, para valores negativos de $t$, o termo $c_{2} v_{1} t e^{\lambda t}$ é novamente dominante, de modo que, quando $t \rightarrow-\infty$, cada trajetória de $\varphi(t)$ é assíntota a uma reta paralela à $v_{1}$. Logo a orientação das trajetórias depende das posições relativas de $v_{1}$ e $v_{2}$. Neste caso, o ponto crítico será chamado nó próprio (atrator ou repulsor) dependendo do sinal de $\lambda$.

Exemplo 1.4. Considere o sistema

$$
\left(\begin{array}{l}
\dot{x} \\
\dot{y}
\end{array}\right)=\left(\begin{array}{cc}
-4 & -1 \\
1 & -6
\end{array}\right)\left(\begin{array}{l}
x \\
y
\end{array}\right)
$$

O único ponto de equilíbrio de (1.8) é a origem (0,0). Os autovalores da matriz característica A de (1.8) são

$$
\lambda_{1}=\lambda_{2}=-5 \text {, }
$$


caracterizando um nó impróprio. Os autovetores associados aos autovalores $\lambda_{1}=\lambda_{2}=3$ são dados, respectivamente, por

$$
v_{1}=(1,1) \text { e } v_{2}=(2,1) .
$$

Portanto a solução geral do sistema (1.8) é da forma

$$
\varphi(t)=c_{1}(1,1) e^{5 t}+c_{2}(1,1) t e^{5 t}+c_{2}(2,1) e^{5 t} .
$$

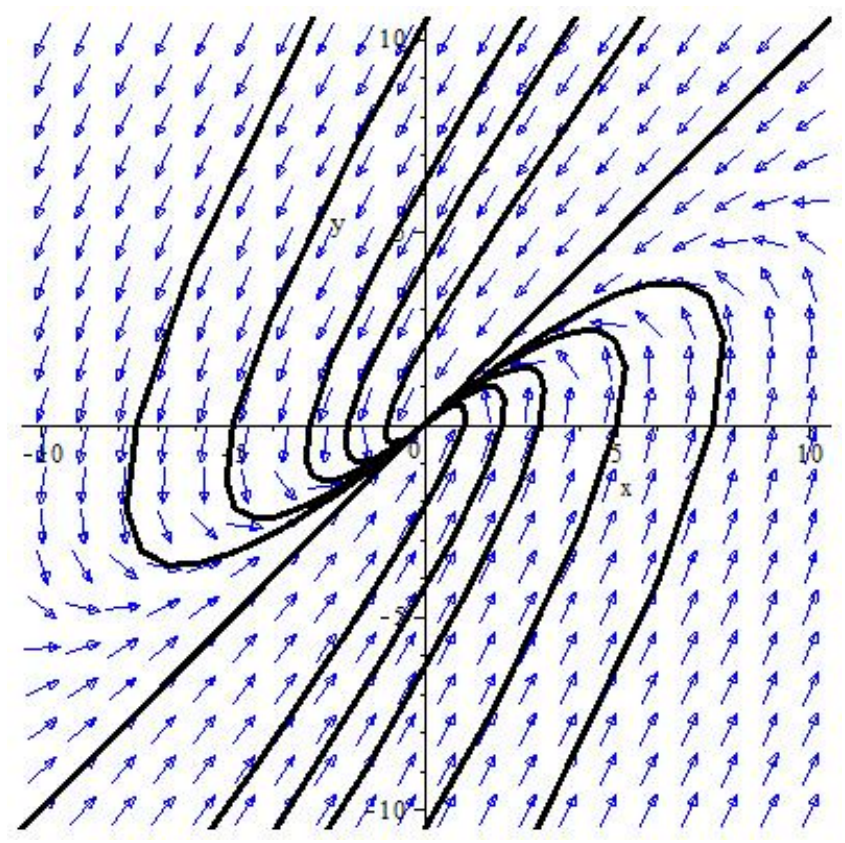

Figura 1.4: Nó impróprio

Caso 4. Os autovalores $\lambda_{1}$ e $\lambda_{2}$ de $A$ são complexos. Considere o sistema

$$
\left\{\begin{array}{c}
\dot{x}=\alpha x+\beta y \\
\dot{y}=-\beta x+\alpha y
\end{array}\right.
$$


onde os autovalores da matriz $A$ são dados por $\lambda_{1}=\alpha+i \beta$ e $\lambda_{2}=\overline{\lambda_{1}}=\alpha-i \beta$. Utilizando a mudança de coordendas polares $r$ e $\theta$,

$$
x=r \cos \theta \quad \text { e } \quad y=r \operatorname{sen} \theta
$$

e derivando as equações de (1.10) em relação a $t$, obtemos

$$
\dot{x}=\dot{r} \cos \theta-r \dot{\theta} \operatorname{sen} \theta=\frac{\dot{r}}{r} x-\dot{\theta} y
$$

$\mathrm{e}$

$$
\dot{y}=\dot{r} \operatorname{sen} \theta+r \dot{\theta} \cos \theta=\frac{\dot{r}}{r} y+\dot{\theta} x .
$$

Portanto $\frac{\dot{r}}{r}=\alpha$ e $\dot{\theta}=-\beta$. Assim, em coordenadas polares, o sistema (1.9) é dado por

$$
\left\{\begin{array}{l}
\dot{r}=\alpha r \\
\dot{\theta}=-\beta
\end{array}\right.
$$

Logo a solução do sistema (1.11) é da forma

$$
\left\{\begin{array}{l}
r(t)=c_{0} e^{\alpha t} \\
\theta(t)=\theta_{0}-\beta t .
\end{array}\right.
$$

Note que a parte real $\alpha$ dos autovalores $\lambda_{1}=\alpha+i \beta$ e $\lambda_{2}=\overline{\lambda_{1}}=\alpha-i \beta$ refere-se ao crescimento ou decrescimento da distância das soluções à origem, enquanto que a parte imaginária $\beta$ destes autovalores refere-se à rotação das soluções em torno da origem. Quando $\alpha=0$, todas as soluções, exceto a solução nula, são elipses. Neste caso, o ponto crítico é chamado centro. Quando $\alpha<0$, as soluções espiralam em direção à origem, então o ponto crítico é denominado foco atrator ou foco estável. Quando $\alpha>0$, as soluções espiralam em sentido oposto à origem, assim o ponto crítico é denominado foco repulsor ou foco instável. 
Note, também, que o sinal de $\beta$ indica o sentido da rotação das soluções em torno da origem, horária caso $\beta<0$ ou anti horária se $\beta>0$. Assim, os autovalores são complexos conjugados, isto é, $\lambda_{1}=\alpha+\beta i$ e $\lambda_{2}=\alpha-\beta i$. A solução geral poderá ser escrita da forma

$$
\varphi(t)=c_{1} \varphi_{1}(t)+c_{2} \varphi_{2}(t)
$$

onde

$$
\begin{aligned}
\varphi_{1}(t) & =e^{\alpha t}\left[v_{1} \cos \beta t-v_{2} \operatorname{sen} \beta t\right] \\
\varphi_{2}(t) & =e^{\alpha t}\left[v_{1} \cos \beta t+v_{2} \operatorname{sen} \beta t\right] .
\end{aligned}
$$

Exemplo 1.5. Considere o sistema

$$
\left(\begin{array}{l}
\dot{x} \\
\dot{y}
\end{array}\right)=\left(\begin{array}{ll}
2 & -5 \\
1 & -2
\end{array}\right)\left(\begin{array}{l}
x \\
y
\end{array}\right) \text {. }
$$

O único ponto de equlíbrio de (1.12) é a origem (0,0), neste caso, denominado centro. Os autovalores da matriz característica $A$ de (1.12) são

$$
\lambda_{1}=i \quad e \quad \lambda_{2}=-i
$$

Logo os autovetores associados a $\lambda_{1}$ e a $\lambda_{2}$ são dados, respectivamente, por

$$
v_{1}=(2+i, 5) \quad \text { e } \quad v_{2}=(2-i, 5) .
$$

Portanto a solução geral do sistema (1.12) é da forma

$$
\varphi(t)=c_{1}(2+i, 5) \cos t+c_{2}(2-i, 5) \operatorname{sen} t
$$

O retrato de fase para o sistema (1.12) é dado pela figura a seguir.

É importante o estudo de sistemas lineares do tipo (1.3), pois é através desta análise que podemos entender localmente o retrato de fase de sistemas não lineares. 


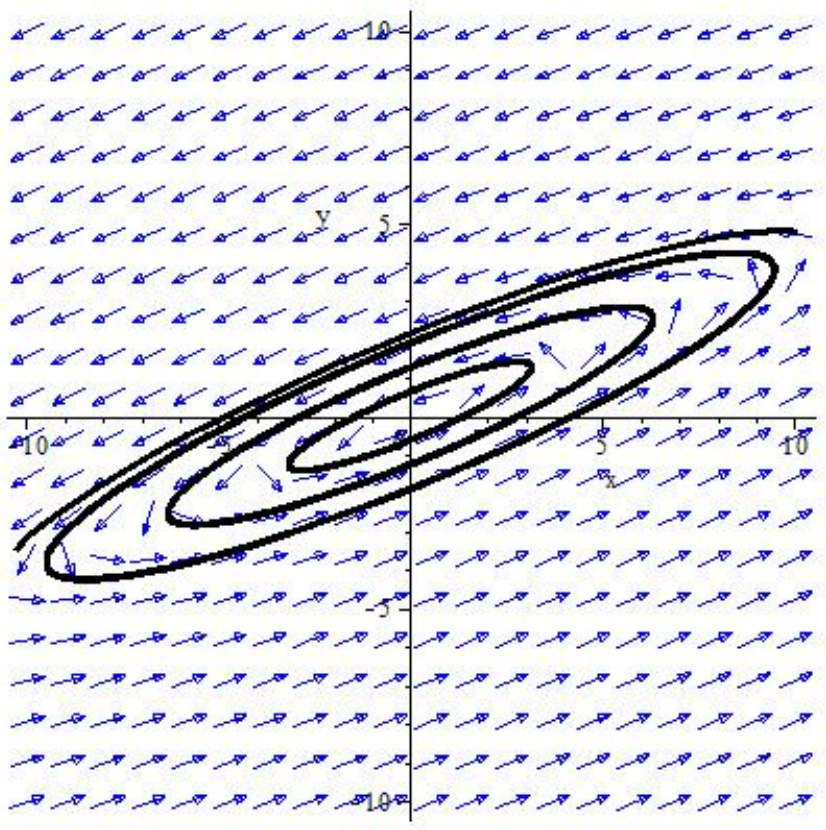

Figura 1.5: Centro 



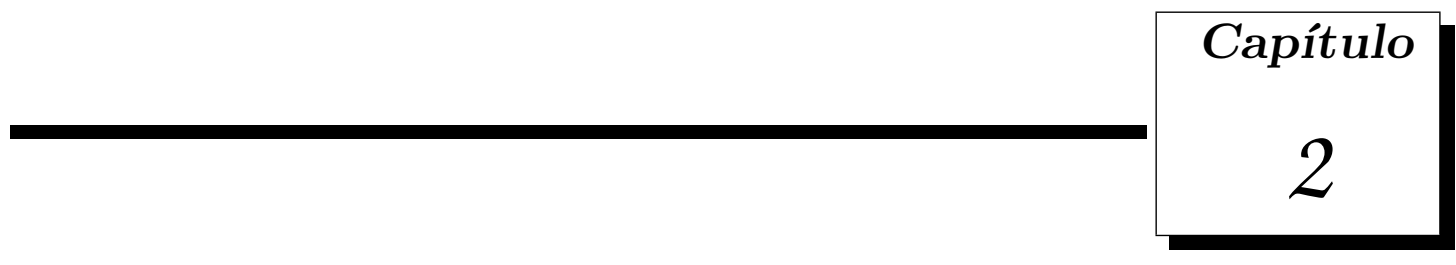

\section{Teoria de bifurcação para EDOs}

Sejam $E, F$ espaços vetoriais reais de dimensão finita. Considere $X \subseteq E$ e $\Lambda \subseteq F$ abertos e $f \in C^{k}(\Lambda \times \mathbb{R} \times X, E)$, para $k \in \mathbb{N}, k \geq 1$, onde $C^{k}(\Lambda \times \mathbb{R} \times X, E)$ denota o espaço das funções de $\Lambda \times \mathbb{R} \times X$ em $E$ que são diferenciáveis até ordem $k$ e que possuem derivadas de ordem $k-1$ contínuas. Neste capítulo, estudaremos bifurcação de soluções perióricas de equações diferenciais dependentes de parâmetro da forma

$$
\dot{x}=f(\lambda, t, x),
$$

onde $f \in C^{k}(\Lambda \times \mathbb{R} \times X, E)$.

Vamos considerar aplicações

$$
\begin{aligned}
T: \Lambda & \rightarrow \mathbb{R}_{+}^{*} \\
\lambda & \mapsto T(\lambda)
\end{aligned}
$$

$\mathrm{e}$

$$
u: \Lambda \times \mathbb{R} \rightarrow E,
$$


e vamos utilizar a notação seguinte: para cada $\lambda \in \Lambda$,

$$
u(\lambda)(t):=u(\lambda, t)
$$

ou seja,

$$
\begin{aligned}
u(\lambda): \mathbb{R} & \rightarrow E \\
t & \mapsto u(\lambda)(t)=u(\lambda, t) .
\end{aligned}
$$

A referência principal para este capítulo é [1].

A seguir, definimos o que vem a ser uma solução periódica de (2.1) e um ponto de bifurcação de uma solução periódica de (2.1).

Definição 2.1. Uma função $u: \Lambda \times \mathbb{R} \rightarrow E$ será dita solução $T(\lambda)$-periódica em relação a $t$ de (2.1), se ela for solução de (2.1) e ainda valer $u(\lambda, t)=u(\lambda, t+T(\lambda))$, para todo $t \in \mathbb{R}$.

Seja $T \in C^{k}\left(\Lambda, \mathbb{R}_{+}^{*}\right)$ e suponha que $u \in C^{k}(\Lambda \times \mathbb{R}, E)$ seja uma solução $T(\lambda)$-periódica da equação diferencial (2.1). Assuma também que a função $f$ do lado direito de (2.1) seja $T(\lambda)$-periódica em relação a $t$. Então a função $\varphi$, definida por

$$
\begin{aligned}
\varphi: \Lambda & \rightarrow B C(\mathbb{R}, E) \\
\lambda & \mapsto \varphi(\lambda)=u(\lambda)
\end{aligned}
$$

onde $u(\lambda): \mathbb{R} \rightarrow E$ pertence a $B C(\mathbb{R}, E)$, onde $B C(\mathbb{R}, E)$ denota o conjunto das funções contínuas e limitadas de $\mathbb{R}$ em $E$. Notemos que a função $\varphi$ descreve o gráfico da solução $u$ de $(2.1)$.

No que segue vamos definir o conceito de ponto de bifurcação de uma solução periódica de $(2.1)$.

Definição 2.2. Com a notação anterior, um par $\left(\lambda_{0}, u\left(\lambda_{0}\right)\right) \in \Lambda \times B C(\mathbb{R}, E)$ será chamado ponto de bifurcação de uma solução periódica $u(\lambda)$ de (2.1), se toda vizinhança de $\left(\lambda_{0}, u\left(\lambda_{0}\right)\right)$ contiver uma solução periódica de (2.1) que não pertence ao gráfico de $u$. 
Antes de estudarmos os problemas de bifurcação para soluções periódicas de equações diferenciais do tipo (2.1), vamos introduzir uma normalização sobre a função $f$ do lado direito de (2.1), a qual denotaremos por $\widetilde{f}$.

Sejam

$$
\widehat{f}(\lambda, t, x):=f(\lambda, t, x+u(\lambda)(t))-f(\lambda, t, u(\lambda)(t))
$$

$\mathrm{e}$

$$
\widetilde{f}(\lambda, t, x):=\left(\frac{T(\lambda)}{2 \pi}\right) \widehat{f}\left(\lambda, \frac{t T(\lambda)}{2 \pi}, x\right)
$$

para todo par $(\lambda, t) \in \Lambda \times \mathbb{R}$, todo $x \in X$, com $X \subseteq E$ aberto e $X \ni 0$.

Afirmação 1. A função $\widetilde{f}$ é $2 \pi$-periódica em $t$ e $\widetilde{f}(\lambda, t, 0)=0$, para todo $\operatorname{par}(\lambda, t) \in \Lambda \times \mathbb{R}$.

Demonstração. É fácil verificarmos que

$$
\widetilde{f}(\lambda, t, 0)=\left(\frac{T(\lambda)}{2 \pi}\right) \widehat{f}\left(\lambda, \frac{t T(\lambda)}{2 \pi}, 0\right)=\frac{T(\lambda)}{2 \pi}[f(\lambda, t, u(\lambda)(t))-f(\lambda, t, u(\lambda)(t))]=0,
$$

para todo $(\lambda, t) \in \Lambda \times \mathbb{R}$. Queremos mostrar que $\tilde{f}$ é $2 \pi$-periódica em $t$, ou seja,

$$
\widetilde{f}(\lambda, t+2 \pi, x)=\tilde{f}(\lambda, t, x), \quad \forall t \in \mathbb{R} .
$$

Utilizando as definições de $\widehat{f}$ e $\widetilde{f}$ e o fato de $f$ ser $T(\lambda)$-periódica em $t$, obtemos

$$
\begin{gathered}
\tilde{f}(\lambda, t+2 \pi, x)=\frac{T(\lambda)}{2 \pi} \widehat{f}\left(\lambda,(t+2 \pi) \frac{T(\lambda)}{2 \pi}, x\right) \\
=\frac{T(\lambda)}{2 \pi}\left[f\left(\lambda,(t+2 \pi) \frac{T(\lambda)}{2 \pi}, x+u(\lambda)(t+2 \pi) \frac{T(\lambda)}{2 \pi}\right)\right. \\
\left.-f\left(\lambda,(t+2 \pi) \frac{T(\lambda)}{2 \pi}, u(\lambda)(t+2 \pi) \frac{T(\lambda)}{2 \pi}\right)\right] \\
=\frac{T(\lambda)}{2 \pi}\left[f\left(\lambda, t \frac{T(\lambda)}{2 \pi}, x+u(\lambda)(t+2 \pi) \frac{T(\lambda)}{2 \pi}\right)-f\left(\lambda, t \frac{T(\lambda)}{2 \pi}, u(\lambda)(t+2 \pi) \frac{T(\lambda)}{2 \pi}\right)\right] \\
=\frac{T(\lambda)}{2 \pi} \widehat{f}\left(\lambda, t \frac{T(\lambda)}{2 \pi}, x\right)=\widetilde{f}(\lambda, t, x)
\end{gathered}
$$


o que completa a prova da Afirmação 1.

Afirmação 2. A aplicação $t \mapsto \widetilde{u}(t)$ será uma solução da equação

$$
\dot{y}=\tilde{f}(\lambda, t, y),
$$

se e somente se,

$$
t \quad \mapsto \quad \widetilde{u}\left(\frac{2 \pi t}{T(\lambda)}\right)+u(\lambda)(t)
$$

for uma solução de

$$
\dot{x}=f(\lambda, t, x) .
$$

Demonstração. Vamos supor que $\widetilde{u}(t)$ seja uma solução da equação

$$
\dot{y}=\tilde{f}(\lambda, t, y) .
$$

Derivando (2.3) em relação a $t$, e utilizando as definições de $\widehat{f}$ e $\widetilde{f}$ respectivamente, obtemos

$$
\begin{gathered}
\frac{2 \pi}{T(\lambda)} \dot{\widetilde{u}}\left(\frac{2 \pi t}{T(\lambda)}\right)+\dot{u}(\lambda)(t)=\frac{2 \pi}{T(\lambda)} \widetilde{f}\left(\lambda, \frac{2 \pi t}{T(\lambda)}, \widetilde{u}\left(\frac{2 \pi t}{T(\lambda)}\right)\right)+f(\lambda, t, u) \\
=\widehat{f}\left(\lambda, t, \widetilde{u}\left(\frac{2 \pi t}{T(\lambda)}\right)\right)+f(\lambda, t, u) \\
=\left[f\left(\lambda, t, \widetilde{u}\left(\frac{2 \pi t}{T(\lambda)}\right)+u(\lambda)(t)\right)-f(\lambda, t, u(\lambda)(t))\right]+f(\lambda, t, u) \\
=f\left(\lambda, t, \widetilde{u}\left(\frac{2 \pi t}{T(\lambda)}\right)+u(\lambda)(t)\right) .
\end{gathered}
$$

Por outro lado, suponhamos que $\widetilde{u}\left(\frac{2 \pi t}{T(\lambda)}\right)+u(\lambda)(t)$ seja uma solução de

$$
\dot{x}=f(\lambda, t, x) .
$$

Logo, a igualdade abaixo é satisfeita, 


$$
\frac{2 \pi}{T(\lambda)} \dot{\widetilde{u}}\left(\frac{2 \pi t}{T(\lambda)}\right)+\dot{u}(\lambda)(t)=f\left(\lambda, t, \widetilde{u}\left(\frac{2 \pi t}{T(\lambda)}\right)+u(\lambda)(t)\right)
$$

Utilizando (2.4) e lembrando que $u$ é solução de $\dot{x}=f(\lambda, t, x)$, obtemos

$$
\begin{gathered}
\frac{2 \pi}{T(\lambda)} \dot{\widetilde{u}}\left(\frac{2 \pi t}{T(\lambda)}\right)=-f(\lambda, t, u)+f\left(\lambda, t, \widetilde{u}\left(\frac{2 \pi t}{T(\lambda)}\right)+u(\lambda)(t)\right) \\
\Longrightarrow \dot{\widetilde{u}}\left(\frac{2 \pi t}{T(\lambda)}\right)=\frac{T(\lambda)}{2 \pi)}\left[-f(\lambda, t, u)+f\left(\lambda, t, \widetilde{u}\left(\frac{2 \pi t}{T(\lambda)}\right)+u(\lambda)(t)\right)\right] \\
=\frac{T(\lambda)}{2 \pi} \widehat{f}\left(\lambda, t, \widetilde{u}\left(\frac{2 \pi t}{T(\lambda)}\right)\right)=\frac{T(\lambda)}{2 \pi} \widehat{f}\left(\lambda, t \frac{2 \pi}{T(\lambda)} \frac{T(\lambda)}{2 \pi}, \widetilde{u}\left(\frac{2 \pi t}{T(\lambda)}\right)\right) \\
=\widetilde{f}\left(\lambda, t \frac{2 \pi}{T(\lambda)}, \widetilde{u}\right) .
\end{gathered}
$$

Logo,

$$
\dot{\widetilde{u}}\left(\frac{2 \pi t}{T(\lambda)}\right)=\tilde{f}\left(\lambda, t \frac{2 \pi}{T(\lambda)}, \widetilde{u}\right)
$$

e, portanto, $\widetilde{u}$ é solução de $\dot{x}=\widetilde{f}(\lambda, t, x)$ e a Afirmação 2 está provada.

No que segue, vamos assumir, sem perda de generalidade, as seguintes condições sobre a função $f$ do lado direito da equação (2.1):

(i) $f: \Lambda \times \mathbb{R} \times X \rightarrow E$ é uma função de classe $C^{k}$, para $k \geq 1$;

(ii) $f$ é $2 \pi$-periódica em $t \in \mathbb{R}$;

(iii) $f(\cdot, \cdot, 0)=0$.

O resultado que enunciaremos agora pode ser encontrado em [1], por exemplo. Sua demonstração não será feita aqui, pois ela depende de vários resultados precedentes. Veja [1], Teorema 25.2.

Teorema 2.3. Suponha que $u\left(\lambda_{0}\right)$ seja uma solução $T\left(\lambda_{0}\right)$-periódica da equação $\dot{x}=f\left(\lambda_{0}, t, x\right)$ e que o problema linearizado

$$
\dot{y}=J f\left(\lambda_{0}, t, u\left(\lambda_{0}\right)(t)\right) y
$$


não possua solução $T\left(\lambda_{0}\right)$-periódica não trivial, onde $J f\left(\lambda_{0}, t, u\left(\lambda_{0}\right)(t)\right)$ denota a matriz jacobiana de $f$ no ponto $\left(\lambda_{0}, t, u\left(\lambda_{0}\right)(t)\right)$. Então existirá uma vizinhança $V$ de $\lambda_{0}$ em $\Lambda$ tal que, para todo $\lambda \in V$, existe uma única solução $T(\lambda)$-periódica $u(\lambda)$ de $\dot{x}=f(\lambda, t, x)$.

O resultado a seguir nos mostra uma relação entre ponto de bifurcação de uma solução de (2.1) e a existência de solução periódica não trivial de equações linearizadas. Tal resultado pode ser encontrado em [1], Proposição 26.1.

Proposição 2.4. Suponha que $\left(\lambda_{0}, 0\right) \in \Lambda \times B C(\mathbb{R}, E)$ seja um ponto de bifurcação da solução $2 \pi$-periódica da equação $\dot{x}=f(\lambda, t, x)$. Então a equação linearizada

$$
\dot{y}=J f\left(\lambda_{0}, t, 0\right) y,
$$

possui solução $2 \pi$-periódica não trivial, onde $J f\left(\lambda_{0}, t, 0\right)$ denota a matriz jacobiana de $f$ no ponto $\left(\lambda_{0}, t, 0\right)$.

Demonstração. Façamos a prova por contradição. Suponha que a equação

$$
\dot{y}=J f\left(\lambda_{0}, t, 0\right) y
$$

não possua solução $2 \pi$-periódica não trivial. Logo, pelo Teorema 2.3 e utilizando a demonstração do mesmo (veja [1], Teorema 25.2), existem vizinhanças $V$ de $\lambda_{0}$ em $\Lambda$ e $U$ de 0 em $B C(\mathbb{R}, E)$ tais que a equação

$$
\dot{x}=f(\lambda, t, x)
$$

possui uma única solução $2 \pi$-periódica $u(\lambda)$ em $U$, para todo $\lambda \in V$. Portanto $u(\lambda)=0$, para todo $\lambda \in V$. Logo $\left(\lambda_{0}, 0\right)$ não é um ponto de bifurcação da equação diferencial (2.1).

Relembremos o leitor que $E$ denota um espaço de Banach de dimensão finita. Considere $X \subseteq E$ um aberto. Por um momento vamos considerar a equação diferencial ordinária sem a dependência do parâmetro $\lambda \in \Lambda$,

$$
\dot{x}=f(x, t)
$$


onde $f \in C^{1}(\mathbb{R} \times X, E)$ e suponha que $u(\cdot, \tau, \xi)$ seja solução maximal do problema de valor inicial (escreveremos PVI)

$$
\left\{\begin{array}{l}
\dot{x}=f(t, x) \\
x(\tau)=\xi .
\end{array}\right.
$$

Vamos denotar o intervalo maximal de existência da solução $u(\cdot, \tau, \xi)$ da seguinte forma

$$
J(\tau, \xi)=\left(t^{-}(\tau, \xi), t^{+}(\tau, \xi)\right) \subseteq \mathbb{R}
$$

Seja $T \in \mathbb{R}$. Vamos definir o operador

$$
\begin{aligned}
& u_{T}: \operatorname{dom}\left(u_{T}\right) \subseteq X \rightarrow X \\
& \xi \mapsto u_{T}(\xi)=u(T, 0, \xi),
\end{aligned}
$$

onde $u(T, 0, \xi)$ é a solução de $\dot{x}=f(t, x)$, em $T \in \mathbb{R}$ e com condição inicial $x(0)=\xi$. O domínio de $u_{T}$, denotado por $\operatorname{dom}\left(u_{T}\right)$, é dado pelo conjunto

$$
\operatorname{dom}\left(u_{T}\right)=\left\{\xi \in X ; t^{-}(0, \xi)<T<t^{+}(0, \xi)\right\}
$$

Notemos que, o operador $u_{T}$ é definido a partir de uma equação diferencial e da condição inicial dada.

Como nosso interesse está em soluções periódicas de equações diferenciais do tipo $\dot{x}=$ $f(t, x)$, é importante obtermos um resultado que nos permite encontrar tais soluções.

O teorema a seguir está presente em [1], Teorema 20.1, e reduz o problema da existência de soluções periódicas da equação diferencial $\dot{x}=f(t, x)$ a encontrar um ponto fixo do operador $u_{T}$.

Teorema 2.5. Sejam $T>0, f \in C^{1}(\mathbb{R} \times X, E)$ uma função T-periódica em $t$, isto é,

$$
f(t+T, x)=f(t, x), \quad t \in \mathbb{R}, x \in X .
$$


Então a equação diferencial ordinária $\dot{x}=f(x, t)$ terá solução T-periódica, se e somente se, o operador $u_{T}: \operatorname{dom}\left(u_{T}\right) \subseteq X \rightarrow X$ possuir ponto fixo.

Demonstração. Seja $u(\cdot, \tau, \xi)$ uma solução $T$-periódica de $\dot{x}=f(x, t)$, com condição inicial $x(\tau)=\xi$. Pela peridiocidade de $u, J(\tau, \xi)=\mathbb{R}$. Sem perda de generalidade, suponha $\tau \leq 0$ e considere $\xi_{0}=u(0, \tau, \xi)$. Notemos que

$$
u\left(t, 0, \xi_{0}\right)=u(t, \tau, \xi)
$$

Utilizando a definição do operator $u_{T}$, a igualdade (2.8) e o fato de $u$ ser $T$-periódica, temos

$$
u_{T}\left(\xi_{0}\right)=u\left(T, 0, \xi_{0}\right)=u(T, \tau, \xi)=u(0, \tau, \xi)=\xi_{0}
$$

Logo $\xi_{0}$ é um ponto fixo de $u_{T}$. Para $\tau \geq 0$, o resultado segue de maneira análoga.

Reciprocamente, seja $\xi \in X$ um ponto fixo de $u_{T}$. Defina $x(t)=u(t+T, 0, \xi)$, para $t \in J(0, \xi) \backslash\{T\}$

Derivando $x(t)$ em relação a $t$ e utilizando o fato de $f$ ser $T$-periódica, obtemos

$$
\dot{x}(t)=\dot{u}(t+T, 0, \xi)=f(t+T, x(t))=f(t, x(t))
$$

e, ainda,

$$
x(0)=u(T, 0, \xi)=u_{T}(\xi)=\xi .
$$

Portanto $x$ é solução do PVI

$$
\left\{\begin{array}{l}
\dot{x}=f(t, x) \\
x(0)=\xi
\end{array}\right.
$$

cuja unicidade de solução implica

$$
u(t, 0, \xi)=u(t+T, 0, \xi) \text {, para todo } t \in J(0, \xi) \backslash\{T\} .
$$

Indutivamente, $u(\cdot, 0, \xi)$ está definida em $t \in \mathbb{R}$ e é solução $T$-periódica de $\dot{x}=f(t, x)$. 
Observação 2.6. Segue da demonstração do Teorema 2.5, que $\xi \in X$ será um ponto fixo de $u_{T}$, se e somente se, $u(\cdot, 0, \xi)$ for uma solução T-periódica de $\dot{x}=f(x, t)$.

Notemos que o Teorema 2.5 é válido se considerarmos a equação diferencial ordinária dependente de parâmetro do tipo (2.1), assim basta tomarmos o operador $u_{T}$ dependente do parâmetro $\lambda \in \Lambda$, definido por $u_{T}(\xi)=(\lambda, T, 0, \xi)$.

Suponha que $u(\lambda, \cdot, 0, \xi)$ seja a solução global do PVI

$$
\left\{\begin{array}{l}
\dot{x}=f(\lambda, t, x) \\
x(0)=\xi
\end{array}\right.
$$

onde $f: \Lambda \times \mathbb{R} \times X \rightarrow E$ é uma função de classe $C^{k}$ normalizada, como fizemos em (2.2), e considere a seguinte função $g: \Lambda \times X \rightarrow E$ definida por

$$
g(\lambda, \xi)=\xi-u(\lambda, 2 \pi, 0, \xi)
$$

onde $u$ é uma solução $2 \pi$-periódica do PVI (2.9). Podemos assumir as seguintes hipóteses:

(i) $g: \Lambda \times X \rightarrow E$ é de classe $C^{k}$;

(ii) $g(\cdot, 0)=0$;

(iii) $\xi_{0}$ será um zero de $g(\lambda, \cdot)$, se e somente se, $u\left(\lambda, \cdot, 0, \xi_{0}\right)$ for uma solução $2 \pi$-periódica de $\dot{x}=f(\lambda, t, x)$.

De fato, suponha que $\xi_{0}$ seja um zero de $g(\lambda, \cdot)$. Logo

$$
g\left(\lambda, \xi_{0}\right)=0 \quad \Longleftrightarrow u\left(\lambda, 2 \pi, 0, \xi_{0}\right)=\xi_{0} \quad \Longleftrightarrow \quad u_{2 \pi}\left(\xi_{0}\right)=\xi_{0}
$$

Portanto $\xi_{0}$ é um ponto fixo do operador $u_{2 \pi}$ similar ao definido em (2.7), mas agora dependente do parâmetro $\lambda \in \Lambda$. Assim, pela Observação $2.6, u\left(\lambda, \cdot, 0, \xi_{0}\right)$ é uma solução $2 \pi$-periódica de $\dot{x}=f(\lambda, t, x)$. 
Portanto o problema de bifurcação para soluções periódicas da equação $\dot{x}=f(\lambda, t, x)$ é equivalente ao problema de bifurcação para soluções da equação dependente de parâmetro $g(\lambda, \xi)=0$.

Ambos os problemas são casos particulares de situações mais gerais, pois o operador $g$ em (2.10) está definido sobre espaços de dimensão finita. A fim de generalizar a definição de ponto de bifurcação, vamos definir um operador sobre espaços de Banach quaisquer.

Sejam $\mathbb{E}, \mathbb{F}$, e $\mathbb{G}$ espaços de Banach quaisquer e considere $\mathbb{X} \subseteq \mathbb{E}, \Lambda \subseteq \mathbb{F}$ subconjuntos abertos, com $0 \in \mathbb{X}$. Defina

$$
\Phi: \Lambda \times \mathbb{X} \rightarrow \mathbb{G}
$$

tal que $\Phi(\lambda, 0)=0$, para todo $\lambda \in \Lambda$.

Definição 2.7. Seja $\Phi: \Lambda \times \mathbb{X} \rightarrow \mathbb{G}$ como definido em (2.11). Um par $\left(\lambda_{0}, 0\right) \in \Lambda \times \mathbb{X}$ será dito um ponto de bifurcação, ou ( $\lambda_{0}$ será chamado um ponto de bifurcação com respeito à solução trivial) da equação $\Phi(\lambda, x)=0$, se toda vizinhança de $\left(\lambda_{0}, 0\right)$ em $\Lambda \times \mathbb{X}$ contiver uma solução $(\lambda, x)$ de $\Phi(\lambda, x)=0$ tal que $x \neq 0$.

Para casos em que $\mathbb{E}, \mathbb{F}$ e $\mathbb{G}$ são espaços de Banach de dimensão finita, poderemos obter resultados para a existência de pontos de bifurcação da equação $\Phi(\lambda, x)=0$, através da Teoria de grau de Brouwer. Faremos uma breve explanação sobre tal teoria, de modo que o leitor esteja apto a entender os próximos resultados no que concerne à bifurcação. Vale lembrar que a Teoria de grau de Brouwer é muito ampla. Aqui, apresentaremos apenas os resultados básicos que serão utilizados neste trabalho.

Sejam $E$ um espaço de Banach de dimensão finita e $\Omega$ um subconjunto aberto e limitado de $E$. O resultado a seguir pode ser encontrado em [1], Teorema 21.5 e trata da existência de uma função chamada grau de Brouwer. A construção de forma detalhada desta função pode ser encontrada em [1] ou [4]. 
Teorema 2.8. Seja E um espaço de Banach de dimensão finita. Então, para todo subconjunto aberto e limitado $\Omega$ de $E$ e todo $y \in E$, existe uma função

$$
\operatorname{deg}(\cdot, \Omega, y): D_{y}(\Omega, E) \rightarrow \mathbb{Z}
$$

chamada grau de Brouwer, onde $D_{y}(\Omega, E)=\{f \in C(\bar{\Omega}, E) ;$ y não pertence a $f(\partial \Omega)\}$, sendo $\partial \Omega$ a fronteira de $\Omega, \bar{\Omega}$ o fecho de $\Omega$ e $\mathbb{Z}$ é o conjunto dos inteiros.

O resultado a seguir pode ser encontrado em [1] e apresenta duas propriedades fundamentais da Teoria de grau de Brouwer, as quais serão utilizadas nos próximos resultados.

Corolário 2.9 ([1], Corolário 21.6). A função grau de Brouwer possui as seguintes propriedades:

(i) (Normalização): Se $y \in \Omega$, então $\operatorname{deg}(I d, \Omega, y)=1$.

(ii) (Invariância Homotópica): Seja $I \subseteq \mathbb{R}$ um intervalo compacto. Assuma que existam uma função $h \in C(\bar{\Omega} \times I, E)$ e $y \in C(I, E)$ satisfazendo

$$
y(\lambda) \text { não pertence a } h(\partial \Omega \times\{\lambda\}), \quad \forall \lambda \in I .
$$

Então,

$$
\operatorname{deg}(h(\cdot, \lambda), \Omega, y(\lambda))
$$

é bem definido e independe de $\lambda \in I$.

A referência para a observação seguinte é [1], pág. 301.

Observação 2.10. Seja $\Omega$ um subconjunto aberto e limitado de um espaço de Banach E, real e de dimensão finita $n$. Se, para cada $f \in C(\bar{\Omega}, E), 0$ não pertencer a $f(\partial \Omega)$. Então

$$
\operatorname{deg}(-f, \Omega, 0)=(-1)^{n} \operatorname{deg}(f, \Omega, 0) .
$$


A seguir, apresentaremos a noção de indexação local, a qual será um caso particular do Teorema 2.8.

Novamente, considere $E$ um espaço de dimensão finita e seja $\Omega \subset E$ aberto e limitado. Considere, também, $f \in C(\bar{\Omega}, E)$ e $x_{0} \in \Omega$ um zero isolado de $f$, ou seja, existe $\epsilon_{0}>0$, tal que $f(x) \neq 0$, para todo $x \in B\left(x_{0}, \epsilon_{0}\right) \backslash\left\{x_{0}\right\}$, onde $B\left(x_{0}, \epsilon_{0}\right)$ denota a bola aberta em $E$ centrada em $x_{0}$ e de raio $\epsilon_{0}$. Então, para todo $\epsilon \in\left(0, \epsilon_{0}\right)$, definimos a função

$$
\begin{aligned}
& \operatorname{deg}(\cdot, \cdot, 0): D_{0}(\Omega, E) \times B\left(x_{0}, \epsilon\right) \rightarrow \mathbb{Z} \\
&(f, x) \quad \mapsto \operatorname{deg}(f, x, 0)
\end{aligned}
$$

a qual depende apenas de $f$ e $x_{0}$. Definimos, também, o conjunto

$$
i_{0}\left(f, x_{0}\right)=\operatorname{deg}\left(f, B\left(x_{0}, \epsilon\right), 0\right), \quad 0<\epsilon<\epsilon_{0},
$$

chamado indexação local de um zero isolado $x_{0}$ de $f$.

O resultado a seguir pode ser encontrado em [1], Proposição 21.8, bem como a sua demonstração e diz que existe uma relação entre a indexação local de um zero isolado da função $f$ e o sinal do determinante do jacobiano de $f$ neste ponto.

Proposição 2.11. Sejam $E$ um espaço de Banach sobre $\mathbb{R}, f \in C(\bar{\Omega}, E)$ e $x_{0} \in \Omega$ tais que $f\left(x_{0}\right)=0$. Suponha que $f$ seja diferenciável em $x_{0}$ e $J f\left(x_{0}\right) \in G L(E)$, onde $G L(E)$ denota o espaço das matrizes invertíveis de $E$ em $E$. Então $x_{0}$ será um zero isolado de $f$ e teremos

$$
i_{0}\left(f, x_{0}\right)=i_{0}\left(J f\left(x_{0}\right), 0\right)=\operatorname{sgn} \operatorname{det}\left[J f\left(x_{0}\right)\right]
$$

Nos próximos resultados deste capítulo, vamos considerar $E$ um espaço de dimensão finita e $X \subset E$ um aberto. Também, vamos considerar $\Lambda \subseteq \mathbb{R}$ e um operador $\Phi \in C(\Lambda \times X, E)$.

O teorema a seguir pode ser encontrado em [1] e diz que se assumirmos certas condições sobre o operador $\Phi$, teremos a existência de um ponto de bifurcação da equação $\Phi(\lambda, x)=0$. 
Teorema 2.12 ([1], Teorema 26.5). Sejam $\Lambda \subseteq \mathbb{R}, \Phi \in C(\Lambda \times X, E)$. Suponha que $\lambda_{0} \in \Lambda$ $e \epsilon>0$ sejam tais que as condições seguintes estejam satisfeitas:

(i) $\Phi(\cdot, 0)=0$;

(ii) Para todo $\lambda \in J:=\left[\lambda_{0}-\epsilon, \lambda_{0}+\epsilon\right] \backslash\left\{\lambda_{0}\right\}$, existe uma vizinhança $J_{\lambda} \times U_{\lambda}$ de $\left(\lambda_{0}, 0\right)$ em $J \times X$ tal que 0 é o único zero de $\Phi(\mu, \cdot)$ em $U_{\lambda}$, para todo $\mu \in J_{\lambda}$.

Então $\left(\lambda_{0}, 0\right)$ será um ponto de bifurcação da equação $\Phi(\lambda, x)=0$, se a indexação local, a qual denotaremos por

$$
i_{\Phi}(\lambda):=i_{0}(\Phi(\lambda, \cdot), 0)
$$

satisfizer $i_{\Phi}\left(\lambda_{0}-\epsilon\right) \neq i_{\Phi}\left(\lambda_{0}+\epsilon\right)$.

Demonstração. Façamos a prova por contradição. Suponha que $\left(\lambda_{0}, 0\right)$ não seja um ponto de bifurcação da equação $\Phi(\lambda, x)=0$. Então para todo $\lambda \in \bar{J}$, existe uma vizinhança $J_{\lambda} \times U_{\lambda}$ de $\left(\lambda_{0}, 0\right) \in \Lambda \times X$ satisfazendo

$$
\Phi(\lambda, x) \neq 0, \text { para todo }(\lambda, x) \in J_{\lambda} \times\left(\bar{U}_{\lambda} \backslash\{0\}\right)
$$

Pela compacidade do fecho $\bar{J}$ de $J$, podemos encontrar um número finito de intervalos $J_{\lambda_{1}}, \ldots, J_{\lambda_{m}}$ que cobrem o intervalo $\bar{J}$.

Considere, também, o conjunto

$$
U=\bigcap_{j=1}^{m} U_{\lambda_{j}} .
$$

Notemos que $U$ é aberto e $0 \in U$. Então $\Phi(\lambda, x)=0$ não possui solução em $\bar{J} \times \bar{U}$, satisfazendo $x \neq 0$.

Portanto,

$$
0 \notin \Phi(\{\lambda\} \times \partial U), \quad \text { para todo } \lambda \in \bar{J}
$$


Portanto, pela propriedade $(i i)$ de invariância homotópica do Corolário 2.9, obtemos

$$
\operatorname{deg}\left(\Phi\left(\lambda_{0}-\epsilon, \cdot\right), U, 0\right)=\operatorname{deg}\left(\Phi\left(\lambda_{0}+\epsilon, \cdot\right), U, 0\right)
$$

Assim, obtemos

$$
\begin{gathered}
i_{\Phi}\left(\lambda_{0}-\epsilon\right)=i_{0}\left(\Phi\left(\lambda_{0}-\epsilon\right), 0\right) \\
=\operatorname{deg}\left(\Phi\left(\lambda_{0}-\epsilon, \cdot\right), U, 0\right)=\operatorname{deg}\left(\Phi\left(\lambda_{0}+\epsilon, \cdot\right), U, 0\right)=i_{0}\left(\Phi\left(\lambda_{0}+\epsilon\right), 0\right)=i_{\Phi}\left(\lambda_{0}+\epsilon\right),
\end{gathered}
$$

o que contradiz a hipótese. Isto conclui a prova.

Podemos encontrar o próximo resultado em [1], Corolário 26.6. Tal resultado nos diz que, sob certas condições para o operador $\Phi$, teremos ponto de bifurcação da equação $\Phi(\lambda, x)=0$.

Corolário 2.13. Sejam $\Lambda \subseteq \mathbb{R} e \Phi \in C^{1}(\Lambda \times X, E)$. Suponha que:

(i) $\Phi(\cdot, 0)=0$;

(ii) Existam $\lambda_{0} \in \Lambda$ e $\epsilon>0$ tais que $\operatorname{Ker}(J \Phi(\lambda, 0))=\{0\}$, para todo $0<\left|\lambda-\lambda_{0}\right| \leq \epsilon$.

Se, além de valerem (i) e (ii), a função

$$
\lambda \in \Lambda \quad \mapsto \quad \operatorname{det}[J \Phi(\lambda, 0)] \in \mathbb{R}
$$

mudar de sinal em $\lambda_{0}$, então $\left(\lambda_{0}, 0\right)$ será um ponto de bifurcação da equação $\Phi(\lambda, x)=0$.

Demonstração. Como $\Phi(\cdot, 0)=0$ e pela hipótese (ii), $J \Phi(\lambda, 0) \in G L(E)$, para todo $\lambda \in \Lambda$, tal que $0<\left|\lambda-\lambda_{0}\right| \leq \epsilon$. Então, pela Proposição 2.11, 0 é um zero isolado de $\Phi(\lambda, \cdot)$ e vale

$$
i_{\Phi}(\lambda)=i_{0}(\Phi(\lambda, \cdot), 0)=i_{0}(J \Phi(\lambda, 0), 0)=\operatorname{sgn} \operatorname{det}[J \Phi(\lambda, 0)]
$$

para todo $\lambda \in \Lambda$, tal que $0<\left|\lambda-\lambda_{0}\right| \leq \epsilon$. Por hipótese, a função $\lambda \mapsto \operatorname{det}[J \Phi(\lambda, 0)]$ muda de sinal em $\lambda_{0}$. Logo $i_{\Phi}\left(\lambda_{0}-\epsilon\right) \neq i_{\Phi}\left(\lambda_{0}+\epsilon\right)$. Então, pelo Teorema 2.12, $\left(\lambda_{0}, 0\right)$ é um ponto de bifurcação de $\Phi(\lambda, x)=0$ e a prova está completa. 
A seguir, mostraremos um exemplo que ilustra o Corolário 2.13, ou seja, mostraremos que, nas condições deste resultado, teremos a existência de ponto de bifurcação, o qual pode ser encontrado em [1].

Exemplo 2.14. Utilizando a notação do Corolário 2.13, sejam $\Lambda=\mathbb{R}, E=\mathbb{R}^{2}$ e considere a equação diferencial ordinária

$$
\dot{x}=f(\lambda, x, y)
$$

onde $f(\lambda, x, y)=\left(\lambda a x-a x^{3}, b y\right)$ e $a, b \in \mathbb{R}^{*}=\mathbb{R} \backslash\{0\}$.

Verifiquemos que (2.12), de fato, satisfaz as condições do Corolário 2.13.

1. Notemos que $f(\lambda, 0,0)=(0,0)$, para todo $\lambda \in \mathbb{R}$. Os zeros de $f(\lambda, \cdot, \cdot)$ são dados por $(0,0) e( \pm \sqrt{\lambda}, 0)$.

2. Calculando a matriz jacobiana de $f$ no ponto $(\lambda, 0,0)$, obtemos

$$
J f(\lambda, 0,0)=\left(\begin{array}{cc}
\lambda a & 0 \\
0 & b
\end{array}\right) .
$$

Assim, se $\lambda \neq 0$, então $\operatorname{Ker}[J f(\lambda, 0,0)]=\{0\}$.

3. Calculando a matriz jacobiana de $f$ no ponto $(\lambda, \pm \sqrt{\lambda}, 0)$, obtemos

$$
J f(\lambda, \pm \sqrt{\lambda}, 0)=\left(\begin{array}{cc}
-2 a \lambda & 0 \\
0 & b
\end{array}\right)
$$

4. A função $\lambda \mapsto \operatorname{det}[J f(\lambda, 0,0)]$, onde $\operatorname{det}[J f(\lambda, 0,0)]=\lambda a b$, muda de sinal em $\lambda=0$. Portanto, pelo Corolário 2.13, $\lambda_{0}=0$ será um ponto de bifurcação em relação à solução trivial da equação $f(\lambda, x, y)=0$. Os autovalores de $J f(\lambda, 0,0)$ são $\lambda a$ e $b$.

Agora, vamos considerar as possíveis condições sobre as constantes $a, b \in \mathbb{R}^{*}$ da matriz jacobiana do item 2 acima. O cálculos dos autovalores para a matriz jacobiana no item 3 pode ser feita de maneira análoga. 
Caso 1: $a, b>0$.

(i) Considere $\lambda<0$. Logo os sinais dos autovalores são $\lambda a<0$ e $b>0$ e temos um ponto de sela.

(ii) Considere $\lambda>0$. Logo os sinais dos autovalores são $\lambda a>0$ e $b>0$ e temos um nó instável.

O retrato de fase de (2.12), para ambas as situações (i) e (ii) do Caso 1 é dado pela Figura 2.1 a seguir.
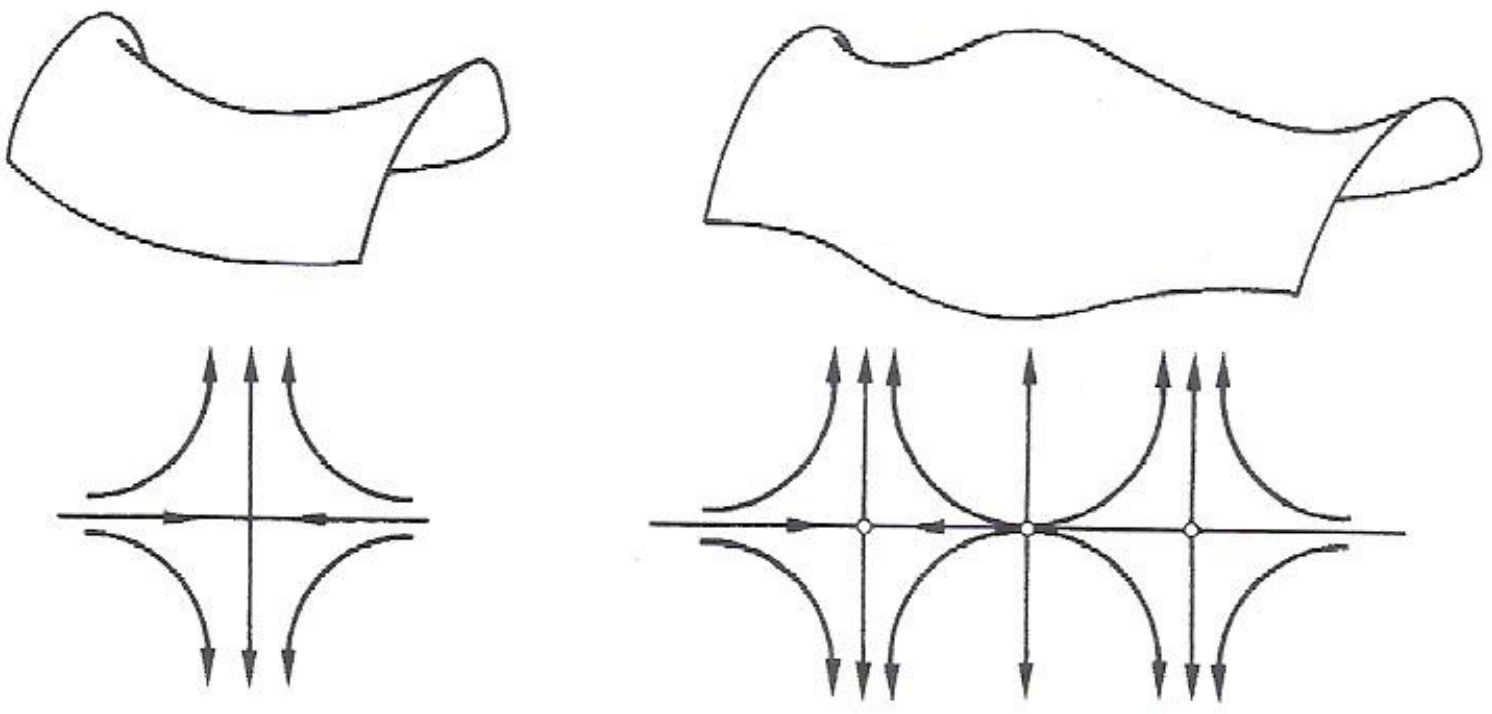

Figura 2.1: Retrato de fase de (2.12) nas condições do Caso 1, itens (i) e (ii) respectivamente.

Associado à equação (2.12), temos o diagrama de bifurcação, o qual nos proporciona uma visão geral do que ocorre com os zeros de (2.12) quando variamos o parâmetro $\lambda \in \mathbb{R}$.

Note que, quando $\lambda$ é negativo, o diagrama de bifurcação de (2.12) (representado pela Figura 2.2 a seguir) é caracterizado pelo ponto de sela. No instante em que $\lambda$ passa por zero, o diagrama de bifurcação de (2.12) torna-se um nó instável, a partir do qual, subdivide-se em duas selas. Neste caso, o diagrama de bifurcação tem a forma de tridente.

Caso 2: $a>0$ e $b<0$. 


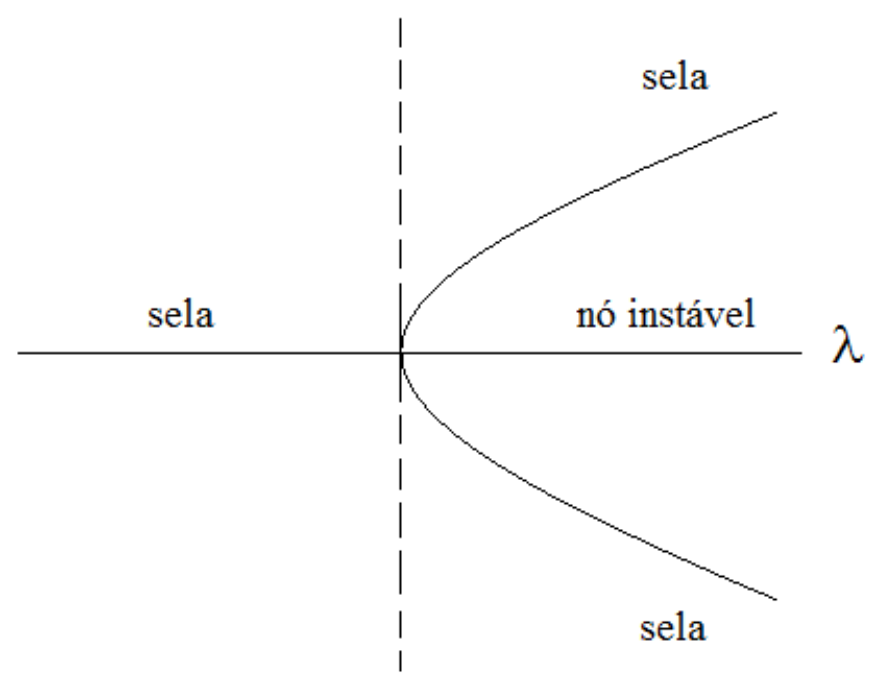

Figura 2.2: Diagrama de bifurcação de (2.12) nas condições do Caso 1.

(i) Considere $\lambda<0$. Logo os sinais dos autovalores de $J f(\lambda, 0,0)$ são dados, respectivamente, por $\lambda a<0$ e $b<0$ e temos um nó atrator.

(ii) Considere $\lambda>0$. Logo os autovalores de $J f(\lambda, 0,0)$ são $\lambda a>0$ e $b<0$ e temos um ponto de sela.

$O$ retrato de fase de (2.12), nas condições do Caso 2 , é dado pela Figura 2.3.

Note que, quando $\lambda$ é negativo, o diagrama de bifurcação de (2.12) (representado pela Figura 2.4 a seguir) é caracterizado pelo nó atrator e, quando $\lambda$ passa por zero, torna-se uma sela, a partir da qual, subdivide-se em dois nós atratores. Neste caso, o diagrama de bifurcação também tem a forma de tridente.

Caso 3: $a<0$ e $b>0$.

(i) Considere $\lambda<0$. Logo os sinais dos autovalores de $J f(\lambda, 0,0)$ são $\lambda a>0$ e $b>0$. Assim, neste caso, $(0,0)$ é um nó instável. 

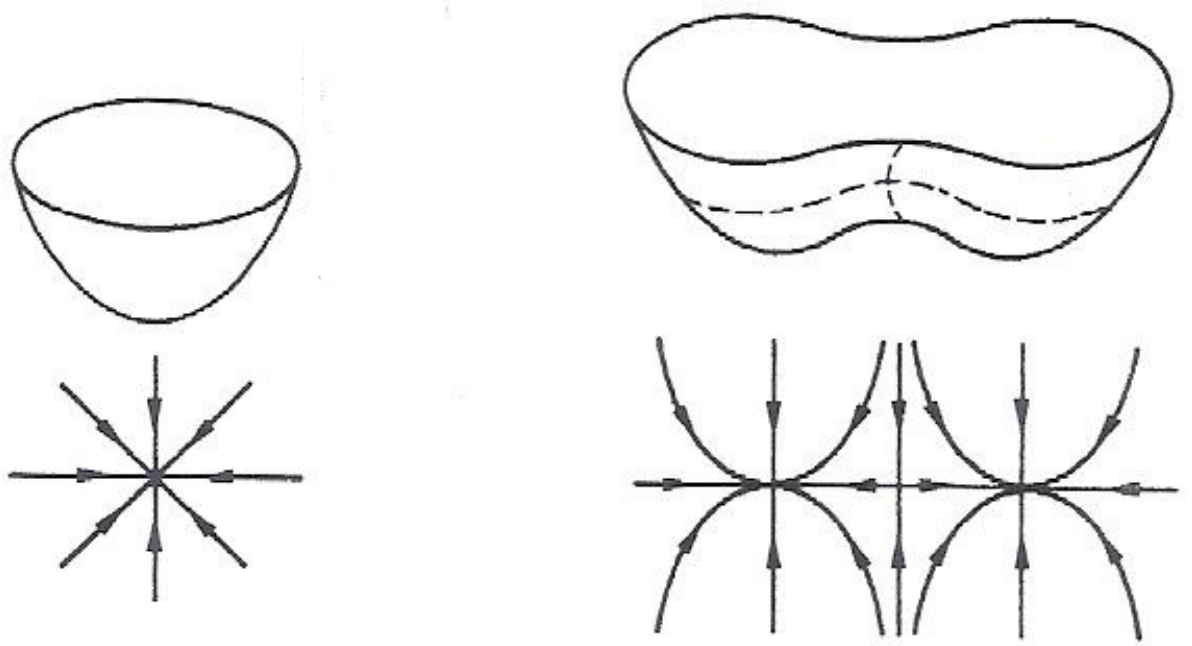

Figura 2.3: Retrato de fase de (2.12) nas condições do Caso 2, itens (i) e (ii) respectivamente.

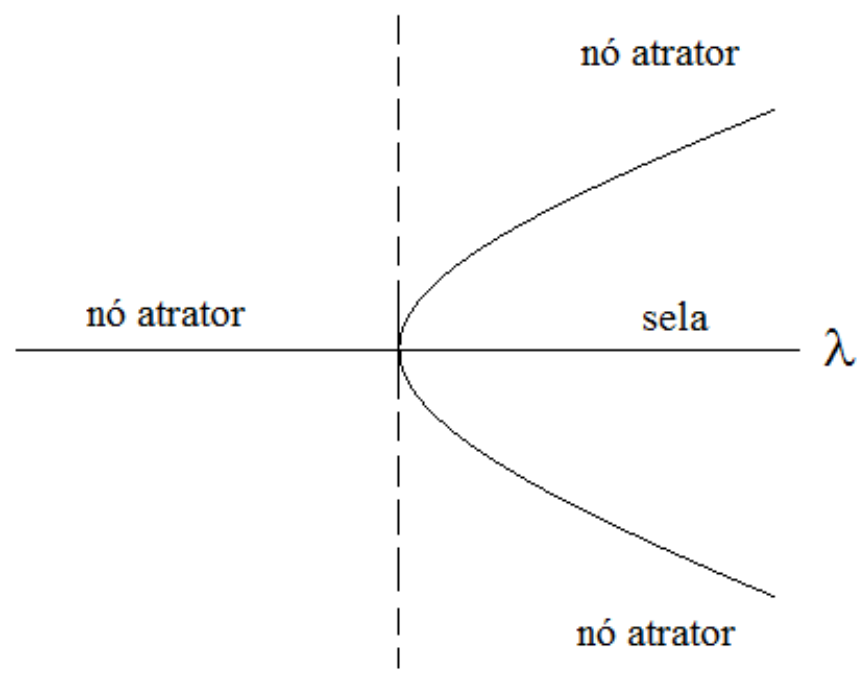

Figura 2.4: Diagrama de bifurcação de (2.12) nas condições do Caso 2.

(ii) Considere $\lambda>0$. Logo os sinais dos autovalores de $J f(\lambda, 0,0)$ são $\lambda a<0$ e $b>0$. Assim, $(0,0)$ é um ponto de sela.

No Caso 3, o retrato de fase de (2.12) são dados pela Figura 2.5. 


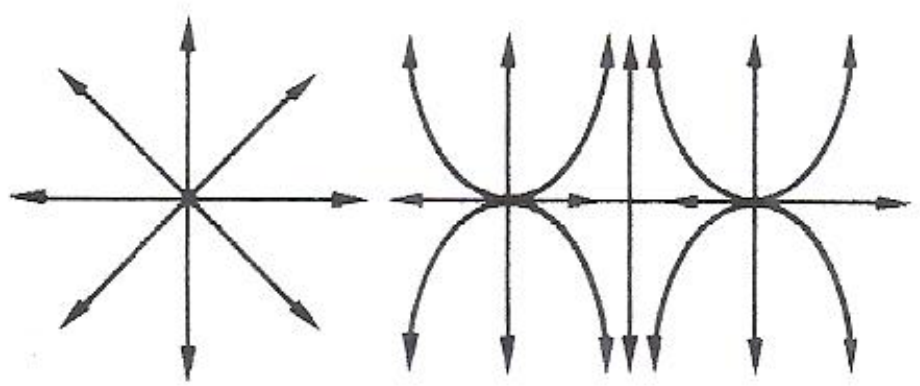

Figura 2.5: Retrato de fase de (2.12) nas condições do Caso 3, itens (i) e (ii) respectivamente.

Note que, quando $\lambda$ é negativo, o diagrama de bifurcação de (2.12) (representado pela Figura 2.6 abaixo) é caracterizado por um nó instável. No instante em que $\lambda$ passa por zero, torna-se uma sela, a partir da qual, subdivide-se em dois nós instáveis.

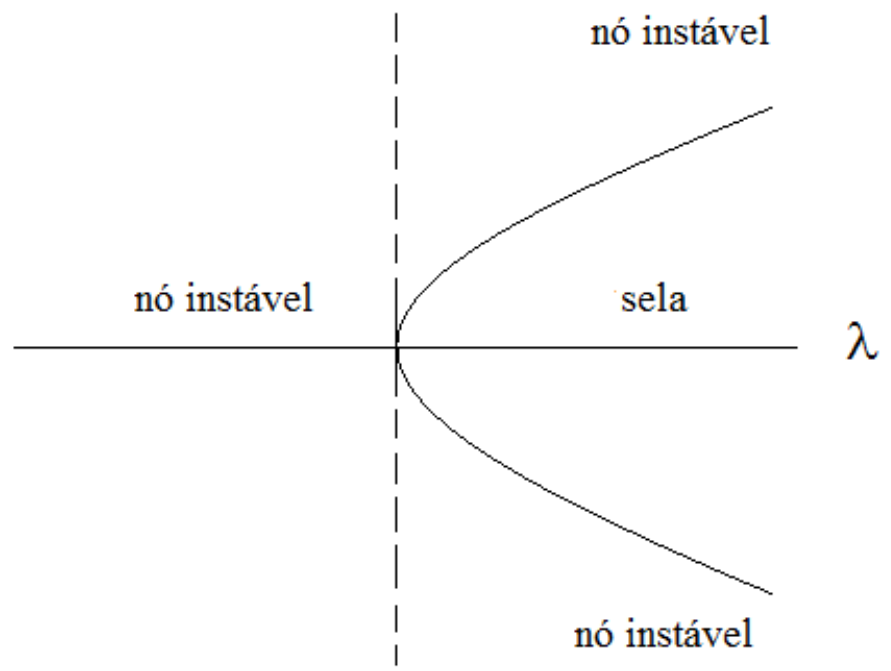

Figura 2.6: Diagrama de bifurcação de (2.12) nas condições do Caso 3. 
Caso 4: $a, b<0$.

(i) Considere $\lambda<0$. Logo os sinais dos autovalores de $J f(\lambda, 0,0)$ são $\lambda a>0$ e $b<0$. Assim, neste caso, $(0,0)$ é um ponto de sela.

(ii) Considere $\lambda>0$. Logo os sinais dos autovalores de $J f(\lambda, 0,0)$ são $\lambda a<0$ e $b<0$. Portanto $(0,0)$ é um nó atrator.

O retrato de fase de (2.12) nas condições do Caso 4 é obtido como no Caso 1, porém inverte-se o sentido das flechas.

Note que, quando $\lambda$ é negativo, o diagrama de bifurcação de (2.12) (representado pela Figura 2.7) é caracterizado por uma sela. No instante em que $\lambda$ passa por zero, o diagrama de bifurcação de (2.12) torna-se um nó atrator, a partir do qual, subdivide-se em duas selas.

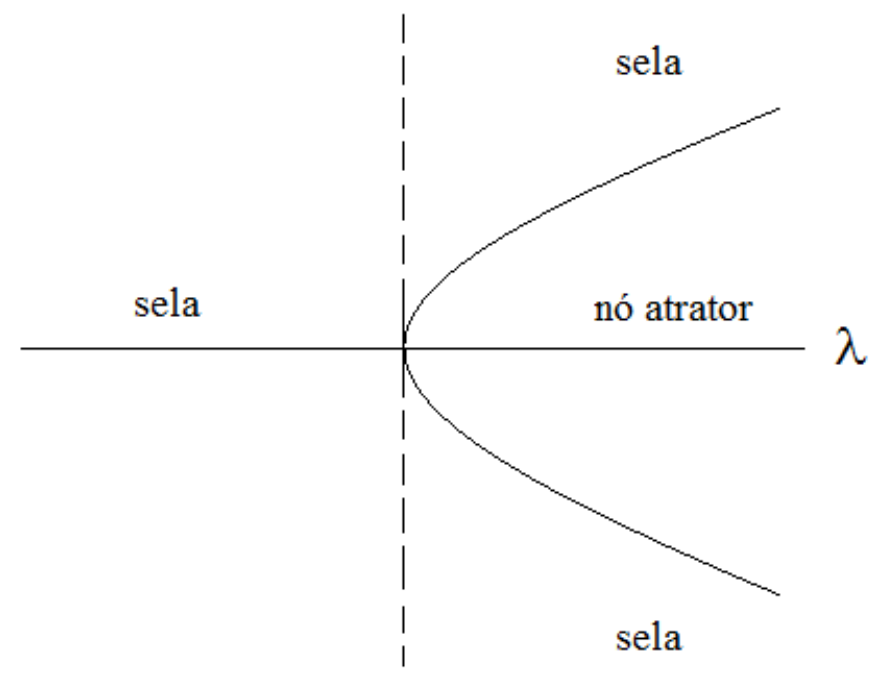

Figura 2.7: Diagrama de bifurcação de (2.12) nas condições do Caso 4. 


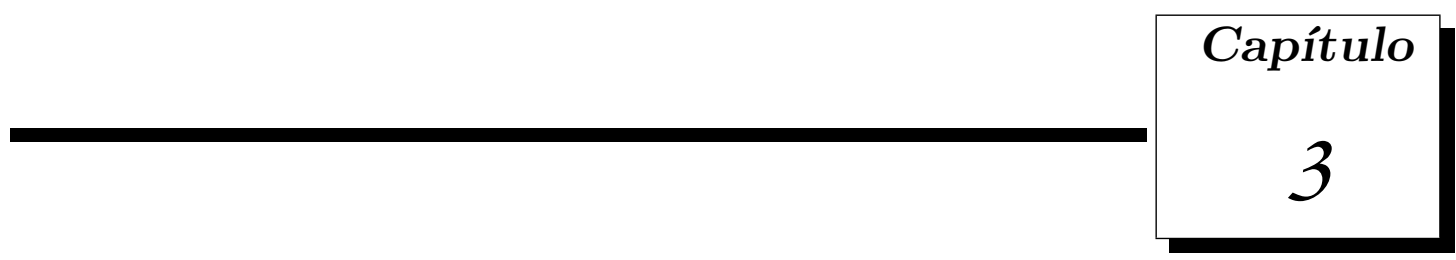

\section{Teoria de bifurcação para EDOs}

\section{generalizadas}

Neste capítulo, apresentaremos a teoria básica das equações diferenciais ordinárias generalizadas, as quais no referiremos simplesmente por EDOGs. Apresentaremos resultados recentes e inéditos: sobre a existência de soluções periódicas e sobre a existência de ponto de bifurcação para soluções periódicas destas equações. Aliás, os conceitos relacionados à teoria de bifurcação para EDOGs são novos, sendo tratados pela primeira vez aqui neste trabalho (e também em [6], que contém os resultados dessa dissertação).

Inicialmente, apresentaremos o conceito, introduzido por Jaroslav Kurzweil em [7] e em [8], de integral de Perron generalizada, hoje conhecida como integral de Kurzweil. Tal conceito permeia toda a teoria de EDOGs.

Denotaremos por $|\cdot|$ e por $\|\cdot\|$ as normas usuais em $\mathbb{R}$ e em $\mathbb{R}^{n}$ respectivamente. 


\subsection{Fundamentos da teoria de EDOGs}

Nesta seção, vamos apresentar os aspéctos fundamentais da teoria das equações diferenciais ordinárias generalizadas (EDOGs) cujas referências básicas são [7], [8] e também o livro $[13]$.

Considere uma função $\delta:[a, b] \rightarrow(0, \infty)$, chamada função calibre de $[a, b]$. Uma divisão marcada do intervalo $[a, b]$ dada pelos pontos $a=t_{0} \leq t_{1} \leq \cdots \leq t_{m}=b$, com as respectivas marcas $\tau_{j} \in\left[t_{j-1}, t_{j}\right]$, para $j=1, \ldots, m$, será chamada $\delta$-fina se tivermos

$$
\left[t_{j-1}, t_{j}\right] \subset\left(\tau_{j}-\delta\left(\tau_{j}\right), \tau_{j}+\delta\left(\tau_{i}\right)\right), \quad i=1, \ldots, m
$$

Denotaremos tal divisão marcada por $d=\left(\tau_{j},\left[t_{j-1}, t_{j}\right]\right)_{j=1}^{|d|}$, onde $|d|=m$.

As próximas definições que apresentaremos foram introduzidos por Jaroslav Kurzweil. Para mais detalhes, veja [13], [7] e [8].

Sejam $T>0$ um número real e $\Lambda \subseteq \mathbb{R}$ um conjunto.

Definição 3.1. Uma função $U: \Lambda \times[0, T] \times[0, T] \rightarrow \mathbb{R}^{n}$ será dita Kurzweil integrável, se existir $I \in \mathbb{R}^{n}$ tal que, para todo $\varepsilon>0$, existe uma função calibre $\delta:[0, T] \rightarrow(0, \infty)$ tal que, para toda divisão marcada $\delta$-fina $d=\left(\tau_{j},\left[t_{j-1}, t_{j}\right]\right)_{j=1}^{|d|}$ de $[0, T]$, temos

$$
\left\|\sum_{j=1}^{|d|}\left[U\left(\lambda, \tau_{j}, t_{j}\right)-U\left(\lambda, \tau_{j}, t_{j-1}\right)\right]-I\right\| \leq \varepsilon .
$$

Neste caso, escrerevemos $I=\int_{0}^{T} D_{s} U(\lambda, \tau, s)$.

Sejam $\mathcal{O} \subset \mathbb{R}^{n}$ um conjunto aberto, $\Lambda \subseteq \mathbb{R}$ um conjunto e $T$ um número real positivo. Considere, também, uma função

$$
F: \Lambda \times \mathcal{O} \times[0, T] \rightarrow \mathbb{R}^{n}
$$




$$
(\lambda, z, t) \mapsto F(\lambda, z, t)
$$

No que segue, apresentaremos a definição de uma solução de um tipo de equação cujo lado direito envolve uma função da forma $F(\lambda, z, t)$ e cuja forma integral possui uma integral como aquela da Definição 3.1 .

Definição 3.2. Uma função $x: \Lambda \times[0, T] \rightarrow \mathbb{R}^{n}$ será dita solução de uma equação diferencial ordinária generalizada da forma

$$
\frac{d x}{d \tau}=D_{t} F(\lambda, x, t)
$$

no intervalo $[0, T]$, se $(\lambda, x(t), t) \in \Lambda \times \mathcal{O} \times[0, T]$ para quaisquer $t \in[0, T]$ e $\lambda \in \Lambda$, e se

$$
x\left(\lambda, s_{2}\right)-x\left(\lambda, s_{1}\right)=\int_{s_{1}}^{s_{2}} D_{t} F(\lambda, x(\lambda, \tau), t)
$$

para todo $s_{1}, s_{2} \in[0, T]$ e todo $\lambda \in \Lambda$.

A integral do lado direito da equação (3.2) deve ser entendida no sentido da Definição 3.1 , onde a função $U: \Lambda \times[0, T] \times[0, T] \rightarrow \mathbb{R}^{n}$ é tal que

$$
U(\lambda, \tau, t)=F(\lambda, x(\lambda, \tau), t)
$$

Para cada $\lambda \in \Lambda$, a equação integral dada em (3.2) é equivalente a

$$
x(\lambda, s)-x(\lambda, 0)=\int_{0}^{s} D_{t} F(\lambda, x(\lambda, \tau), t), \quad s \in[0, T]
$$

Um caso particular da Definição 3.1 é apresentado a seguir e pode ser encontrado em $[13]$.

Definição 3.3. Uma função $U:[0, T] \times[0, T] \rightarrow \mathbb{R}^{n}$, onde $T>0$, será dita Kurzweil integrável se, para todo $\varepsilon>0$, existir uma função calibre $\delta:[0, T] \rightarrow(0, \infty)$ tal que, para 
toda divisão marcada $\delta$-fina $d=\left(\tau_{j},\left[t_{j-1}, t_{j}\right]\right)_{j=1}^{|d|}$ de $[0, T]$, temos

$$
\left\|\sum_{j=1}^{|d|}\left[U\left(\tau_{j}, t_{j}\right)-U\left(\tau_{j}, t_{j-1}\right)\right]-I\right\| \leq \varepsilon .
$$

Neste caso, escreveremos $I=\int_{0}^{T} D U(\tau, s)$.

Sejam $B_{R} \subset \mathbb{R}^{n}$ a bola aberta centrada na origem e de raio $R>0, T$ um número real positivo e considere uma seguinte função

$$
\begin{aligned}
F: \quad B_{R} \times[0, T] & \rightarrow \mathbb{R}^{n} \\
(z, t) & \mapsto F(z, t) .
\end{aligned}
$$

Com esta notação, mencionamos a definição abaixo.

Definição 3.4. Uma função $x:[0, T] \rightarrow \mathbb{R}^{n}$ será dita uma solução da equação diferencial ordinária generalizada

$$
\frac{d x}{d \tau}=D F(x, t)
$$

em $[0, T] \subset \mathbb{R}$, se $(x(t), t) \in B_{R} \times[0, T]$, para todo $t \in[0, T]$, e se tivermos

$$
x\left(s_{2}\right)-x\left(s_{1}\right)=\int_{s_{1}}^{s_{2}} D F(x(\tau), t)
$$

para quaisquer $s_{1}, s_{2} \in[0, T]$.

Note que a integral do lado direito da equação (3.5) deve ser entendida no sentido da Definição 3.3, tomando-se $U:[0, T] \times[0, T] \rightarrow \mathbb{R}^{n}$ como sendo

$$
U(\tau, t)=F(x(\tau), t)
$$

Vamos considerar $\Omega=B_{R} \times[0, T]$, onde $B_{R} \subset \mathbb{R}^{n}$ é a bola aberta centrada na origem e de raio $R>0$ e $T>0$. Sejam, também, $h_{R}:[0, T] \rightarrow \mathbb{R}$ uma função não decrescente e contínua 
à esquerda e $\omega_{R}:[0,+\infty) \rightarrow \mathbb{R}$ uma função contínua, crescente e que satisfaz $\omega_{R}(0)=0$. Utilizando a mesma notação como na referência [13], denotaremos por $\mathcal{F}(\Omega, h, \omega)$ a classe das funções $F: B_{R} \times[0, T] \rightarrow \mathbb{R}^{n}$ para as quais as seguintes desigualdades são satisfeitas:

$$
\begin{gathered}
\left\|F\left(z, t_{2}\right)-F\left(z, t_{1}\right)\right\| \leq\left|h_{R}\left(t_{2}\right)-h_{R}\left(t_{1}\right)\right| \\
\left\|F\left(z, t_{2}\right)-F\left(z, t_{1}\right)-F\left(y, t_{2}\right)+F\left(y, t_{1}\right)\right\| \leq \omega_{R}(\|z-y\|)\left|h_{R}\left(t_{2}\right)-h_{R}\left(t_{1}\right)\right|,
\end{gathered}
$$

para quaisquer $z, y \in B_{R}$ e quaisquer $t_{1}, t_{2} \in[0, T]$.

No que segue, denotaremos por $B V$ o espaço das funções de variação limitada $x:[0, T] \rightarrow$ $\mathbb{R}^{n}$, com a norma da variação dada por

$$
\|x\|_{B V}=\|x(0)\|+\operatorname{var}_{0}^{T} x
$$

onde $\operatorname{var}_{0}^{T}=\sup _{d} \sum_{i=1}^{|d|}\left\|x\left(t_{i}\right)-x\left(t_{i-1}\right)\right\|$ denota a variação de $x$ em $[0, T]$ e o supremo é tomado sobre todas as divisões $d=\left(\left[t_{i}, t_{i-1}\right]\right)_{i=1}^{|d|}$ de $[0, T]$. Sabe-se que $\left(B V,\|\cdot\|_{B V}\right)$ é um espaço de Banach.

O resultado a seguir pode ser encontrado em [13] e nos diz que se sob certas condições, podemos majorar a integral no sentido da Definição 3.3 pela função $h_{R}$ definida acima.

Lema 3.5 ([13], Lema 3.9). Seja $\Omega=B_{R} \times[0, T]$ e suponha que $F: \Omega \rightarrow \mathbb{R}^{n}$ satisfaça a condição (3.6). Se $x:[0, T] \rightarrow \mathbb{R}^{n}$, com $(x(t), t) \in \Omega$, para todo $t \in[0, T]$, e se a integral $\int_{s_{1}}^{s_{2}} D F(x(\tau), t)$ existir, então para quaisquer $s_{1}, s_{2} \in[0, T]$, teremos

$$
\left\|\int_{s_{1}}^{s_{2}} D F(x(\tau), t)\right\| \leq\left|h_{R}\left(s_{2}\right)-h_{R}\left(s_{1}\right)\right| .
$$

O próximo resultado está presente em [13] e nos diz que, se $F$ satisfizer a condição (3.6) e se $x$ for uma solução da EDOG (3.4), então $x$ será de variação limitada.

Teorema 3.6 ([13], Corolário 3.11). Suponha que a função $F: B_{R} \times[0, T] \rightarrow \mathbb{R}^{n}$ satisfaça a condição (3.6). Se $x:[0, T] \rightarrow \mathbb{R}^{n}$ for uma solução da EDOG (3.4), então x será de 
variação limitada em $[0, T]$, com

$$
{ }_{0}^{T} x \leq h_{R}(T)-h_{R}(0)<+\infty
$$

O teorema abaixo também pode ser encontrado em [13] e nos diz que, sob certas condições, teremos a existência da integral no sentido da Definição 3.3.

Teorema 3.7 ([13], Corolário 3.16). Considere $F \in \mathcal{F}\left(\Omega, h_{R}, \omega_{R}\right)$ e suponha que $x:[0, T] \rightarrow$ $\mathbb{R}^{n}$ seja uma função de variação limitada tal que $(x(s), s) \in B_{R} \times[0, T]$, para todo $s \in[0, T]$. Então a integral $\int_{0}^{T} D F(x(\tau), t)$ existe.

O lema abaixo pode ser encontrado em [10], bem como a sua demonstração. Tal lema apresenta uma importante propriedade sobre a classe das funções $\mathcal{F}\left(\Omega, h_{R}, \omega_{R}\right)$ e será um resultado muito útil nas demonstrações deste capítulo.

Lema 3.8 ([10], Lema 5). Suponha que $F: \Omega \rightarrow \mathbb{R}^{n}$ pertença à classe $\mathcal{F}\left(\Omega, h_{R}, \omega_{R}\right)$. Se $x, y:[0, T] \rightarrow B_{R}$ forem funções de variação limitada, então

$$
\left\|\int_{0}^{T} D[F(x(\tau), t)-F(y(\tau), t)]\right\| \leq \int_{0}^{T} \omega_{R}(\|x(t)-y(t)\|) d h_{R}(t) .
$$

\subsection{Soluções periódicas de EDOGs}

Nesta seção, nosso objetivo é introduzir o conceito de soluções periódicas de equações diferencias ordinárias generalizadas, bem como provar um resultado de existência deste tipo de solução.

Definição 3.9. Seja $T$ um número real positivo. Diremos que $x:[0, T] \rightarrow \mathbb{R}^{n}$ é uma solução T-periódica de uma EDOG

$$
\frac{d x}{d \tau}=D F(x, t)
$$

se $x$ for solução de (3.8) e, se além disso, tivermos $x(0)=x(T)$. 
No que segue, vamos introduzir um operador $\mathcal{M}$ dado a seguir:

$$
\begin{aligned}
\mathcal{M}:[0,1] \times B V & \rightarrow B V \\
(\lambda, x) & \mapsto \mathcal{M}(\lambda, x)
\end{aligned}
$$

tal que, para cada $s \in[0, T]$,

$$
\mathcal{M}(\lambda, x)(s)=x(0)+\int_{0}^{T} D F(x(\tau), t)+\lambda \int_{0}^{s} D F(x(\tau), t) .
$$

Segue do Teorema 3.7, que o operador $\mathcal{M}$ está bem definido.

O próximo resultado pode ser encontrado em [6] e nos diz que se $F \in \mathcal{F}\left(\Omega, h_{R}, \omega_{R}\right)$, então o operador $\mathcal{M}$ será contínuo com relação à segunda variável.

Teorema 3.10. Seja $F \in \mathcal{F}\left(\Omega, h_{R}, \omega_{R}\right)$. Então o operador $\mathcal{M}:[0,1] \times B V \rightarrow B V$ será contínuo com relação à segunda variável, onde $\mathcal{M}$ é dado por (3.9).

Demonstração. Sejam $x, y$ soluções da EDOG (3.8) tais que $x(s), y(s) \in B_{R}$, para todo $s \in[0, T]$. Logo $x, y \in B V$, pelo Teorema 3.6. Então,

$$
\begin{aligned}
& \|\mathcal{M}(\lambda, y)-\mathcal{M}(\lambda, x)\|_{B V}=\|\mathcal{M}(\lambda, y)(0)-\mathcal{M}(\lambda, x)(0)\|+_{0}^{T}[\mathcal{M}(\lambda, y)-\mathcal{M}(\lambda, x)] \\
\leq & \|y(0)-x(0)\|+\left\|\int_{0}^{T}[D F(y(\tau), t)-D F(x(\tau), t)]\right\|+_{0}^{T}[\mathcal{M}(\lambda, y)-\mathcal{M}(\lambda, x)] .
\end{aligned}
$$

Mas, pelo Lema 3.8,

$$
\left\|\int_{0}^{T} D[F(y(\tau), t)-D F(x(\tau), t)]\right\| \leq \int_{0}^{T} \omega_{R}(\|y(t)-x(t)\|) d h_{R}(t) .
$$

Então,

$$
\|\mathcal{M}(\lambda, y)-\mathcal{M}(\lambda, x)\|_{B V} \leq\|x(0)-y(0)\|+\int_{0}^{T} \omega_{R}(\|y(t)-x(t)\|) d h_{R}(t)+
$$




$$
+_{0}^{T}[\mathcal{M}(\lambda, y)-\mathcal{M}(\lambda, x)]
$$

Seja uma divisão arbitrária $d=\left(\left[t_{i-1}, t_{i}\right]\right)_{i=1}^{|d|}$ de $[0, T]$. Utilizando $(3.11)$, obtemos

$$
\begin{gathered}
\sum_{i=1}^{|d|}\left\|\mathcal{M}(\lambda, y)\left(t_{i}\right)-\mathcal{M}(\lambda, x)\left(t_{i}\right)-\mathcal{M}(\lambda, y)\left(t_{i-1}\right)+\mathcal{M}(\lambda, x)\left(t_{i-1}\right)\right\|= \\
\sum_{i=1}^{|d|}\left\|\lambda \int_{t_{i-1}}^{t_{i}} D F(y(\tau), t)-\lambda \int_{t_{i-1}}^{t_{i}} D F(x(\tau), t)\right\| \\
\leq \lambda \sum_{i=1}^{|d|}\left\|\int_{t_{i-1}}^{t_{i}} D[F(x(\tau), t)-F(y(\tau), t)]\right\| \leq \lambda \int_{0}^{T} \omega(\|y(t)-x(t)\|) d h_{R}(t) .
\end{gathered}
$$

Tomando o supremo sobre todas as divisões $d=\left(\left[t_{i-1}, t_{i}\right]\right)_{i=1}^{|d|}$ de $[0, T]$, obtemos

$$
{ }_{0}^{T}[\mathcal{M}(\lambda, y)-\mathcal{M}(\lambda, x)] \leq \lambda \int_{0}^{T} \omega(\|y(t)-x(t)\|) d h(t)
$$

Utilizando a última desigualdade e (3.10), obtemos

$$
\begin{aligned}
\| \mathcal{M}(\lambda, y) & -\mathcal{M}(\lambda, x)\left\|_{B V} \leq\right\| x(0)-y(0) \|+(1+\lambda) \int_{0}^{T} \omega_{R}(\|y(t)-x(t)\|) d h_{R}(t) \\
& \leq\|x(0)-y(0)\|+(1+\lambda) \omega_{R}\left(\sup _{t \in[0, T]}\|y(t)-x(t)\|\right) \int_{0}^{T} d h_{R}(t) \\
& \leq\|x(0)-y(0)\|+(1+\lambda) \omega_{R}\left(\|y-x\|_{B V[0, T]}\right)\left[h_{R}(T)-h_{R}(0)\right]
\end{aligned}
$$

e utilizando o fato que

$$
\sup _{t \in[0, T]}\|y(t)-x(t)\| \leq\|y-x\|_{B V[0, T]},
$$

então o operador $\mathcal{M}$ será contínuo em $B V$ e a prova está completa.

Agora, vamos considerar a função $F: B_{R} \times[0, T] \rightarrow \mathbb{R}^{n}$ e a EDOG

$$
\frac{d x}{d \tau}=D F(x, t)
$$


O resultado seguinte descreve a correspondência entre soluções periódicas de (3.12) e os ponto fixos do operador $\mathcal{M}$ definido em (3.9), para $\lambda=1$, isto é,

$$
\mathcal{M}(1, x)(s)=x(0)+\int_{0}^{T} D F(x(\tau), t)+\int_{0}^{s} D F(x(\tau), t)
$$

para todo $x \in B V$ e todo $s \in[0, T]$.

Teorema 3.11. Uma função $x:[0, T] \rightarrow \mathbb{R}^{n}$ será uma solução T-periódica de (3.12), se e somente se, $x$ for um ponto fixo do operador $\mathcal{M}: B V \rightarrow B V$, onde $\mathcal{M}$ é definido por (3.13), ou seja, $\mathcal{M}(x)(s)=x$, para todo $s \in[0, T]$.

Demonstração. Suponha que $x$ seja uma solução $T$-periódica de (3.12). Isso significa que $x$ é de variação limitada (pelo Teorema 3.6) e que, para todo $s \in[0, T]$, vale

$$
x(s)=x(0)+\int_{0}^{s} D F(x(\tau), t) .
$$

Em particular,

$$
x(T)=x(0)+\int_{0}^{T} D F(x(\tau), t) .
$$

Como por hipótese $x(T)=x(0)$, obtemos

$$
\int_{0}^{T} D F(x(\tau), t)=0
$$

Logo, para todo $s \in[0, T]$, temos

$$
x(s)=x(0)+\int_{0}^{T} D F(x(\tau), t)+\int_{0}^{s} D F(x(\tau), t)
$$

o que implica que

$$
\mathcal{M}(x)(s)=x(s), \quad s \in[0, T]
$$

Reciprocamente, seja $x \in B V$ tal que $\mathcal{M}(x)(s)=x(s)$, para todo $s \in[0, T]$. 
Tomando $s=0$ em (3.30), obtemos

$$
\int_{0}^{T} D F(x(\tau), t)=0
$$

e, tomando $s=T$ em (3.30) e utilizando (3.16), obtemos

$$
x(T)-x(0)=2 \int_{0}^{T} D F(x(\tau), t)=0
$$

ou seja, $x(T)=x(0)$. Mas, por hipótese, para cada $x \in B V$, temos

$$
\mathcal{M}(x)(s)=x(0)+\int_{0}^{T} D F(x(\tau), t)+\int_{0}^{s} D F(x(\tau), t)=x(s), \quad s \in[0, T] .
$$

Assim, usando a equação (3.16), obtemos, para todo $s \in[0, T]$, a igualdade seguinte

$$
x(s)-x(0)=\int_{0}^{s} D F(x(\tau), t)
$$

o que significa que $x$ é uma solução (T-periódica) de (3.12).

O próximo resultado pode ser encontrado em [6] e nos diz que, se $F$ pertencer à classe $\mathcal{F}\left(\Omega, h_{R}, \omega_{R}\right)$, então o operador $\mathcal{M}:[0,1] \times B V \rightarrow B V$ como definido em (3.9) será compacto.

Lema 3.12. Seja $F \in \mathcal{F}\left(\Omega, h_{R}, \omega_{R}\right)$. Então o operador $\mathcal{M}$, como definido em (3.9), leva conjuntos limitados de $[0,1] \times B V$ em conjuntos relativamente compactos de $B V$.

Demonstração. Basta mostrarmos que, se $\left\{\lambda_{n}, x_{n}\right\}_{n \in \mathbb{N}}$ for uma sequência limitada em $[0,1] \times$ $B V$, então $\left\{\mathcal{M}\left(\lambda_{n}, x_{n}\right)\right\}_{n \in \mathbb{N}}$ possuirá uma subsequência convergente em $B V$.

Suponha que $\left\{\lambda_{n}, x_{n}\right\}_{n \in \mathbb{N}}$ seja uma sequência limitada em $[0,1] \times B V$. Então, pelo Teorema da Escolha de Helly (veja [11]), existe uma subsequência $\left\{x_{n_{k}}\right\}_{k \in \mathbb{N}}$ de $\left\{x_{n}\right\}_{n \in \mathbb{N}}$, que converge pontualmente em $[0, T]$ para uma função $y \in B V$. Como $\left\{\lambda_{n}\right\}_{n \in \mathbb{N}}$ é uma sequência limitada em $\mathbb{R}$, segue do Teorema de Bolzano-Weierstrass a existência de uma subsequência $\left\{\lambda_{n_{k}}\right\}_{k \in \mathbb{N}}$ de $\left\{\lambda_{n}\right\}_{n \in \mathbb{N}}$ que converge uniformemente em $[0,1]$ para $\lambda$. Sem perda de generalidade, podemos denotar estas subsequências por $\left\{x_{n}\right\}_{n \in \mathbb{N}}$ e $\left\{\lambda_{n}\right\}_{n \in \mathbb{N}}$ respectivamente. 
Usando as mesmas ideias como na prova do Teorema 3.10 e usando o Lema 3.5, obtemos

$$
\begin{gathered}
\left\|\mathcal{M}\left(\lambda_{n}, x_{n}\right)-\mathcal{M}(\lambda, y)\right\|_{B V} \leq\left\|x_{n}(0)-y(0)\right\| \\
+\left\|\lambda_{n} \int_{0}^{T} D F\left(x_{n}(\tau), t\right)-\lambda \int_{0}^{T} D F(y(\tau), t)\right\| \\
\leq\left\|x_{n}(0)-y(0)\right\|+\lambda_{n}\left\|\int_{0}^{T}\left[D F\left(x_{n}(\tau), t\right)-\int_{0}^{T} D F(y(\tau), t)\right]\right\|+ \\
\quad+\left|\lambda_{n}-\lambda\right|\left\|\int_{0}^{T} D F(y(\tau), t)\right\| \\
\leq\left\|x_{n}(0)-y(0)\right\|+\lambda_{n} \omega_{R}\left(\sup _{t \in[0, T]}\left\|x_{n}(t)-y(t)\right\|\right) \int_{0}^{T} d h_{R}(t)+\left|\lambda_{n}-\lambda\right|\left[h_{R}(T)-h_{R}(0)\right] \\
\leq\left\|x_{n}(0)-y(0)\right\|+\left[\lambda_{n} \omega_{R}\left(\sup _{t \in[0, T]}\left\|x_{n}(t)-y(t)\right\|\right)+\left|\lambda_{n}-\lambda\right|\right]\left[h_{R}(T)-h_{R}(0)\right] .
\end{gathered}
$$

É fácil verificarmos que o lado direito da desigualdade acima tende para zero, quando $n \rightarrow \infty$. Portanto o resultado segue.

\subsection{Existência de soluções periódicas de EDOGs}

Nesta seção, vamos utilizar as notações e terminologias introduzidas anteriormente e nosso objetivo será garantir a existência de pelo menos uma solução $T$-periódica da EDOG

$$
\frac{d x}{d \tau}=D F(x, t)
$$

onde $F \in \mathcal{F}\left(\Omega, h_{R}, \omega_{R}\right)$. Faremos isso utilizando a teoria do grau coincidente (veja [5]). Vamos utilizar o Teorema 2.8 (veja o Capítulo 2), onde definimos a função grau de Brouwer no caso particular em que $y=0$.

Seja $B \subset \mathbb{R}^{n}$ um conjunto aberto e limitado tal que $0 \in B$. Considere, também, o conjunto das funções contínuas $f: \bar{B} \subset \mathbb{R}^{n} \rightarrow \mathbb{R}^{n}$ tais que $0 \notin f(\partial B)$, denotado por 
$\mathbf{D}_{0}\left(B, \mathbb{R}^{n}\right)$. A função grau de Brouwer de $f$ em $B$ é dada por

$$
\begin{aligned}
\operatorname{deg}(\cdot, B, 0): \mathbf{D}_{0}\left(B, \mathbb{R}^{n}\right) & \rightarrow \mathbb{Z} \\
f & \mapsto \operatorname{deg}(f, B, 0) .
\end{aligned}
$$

onde $\bar{B}$ e $\partial B$, denotam, respectivamente, o fecho e a fronteira do conjunto $B$.

Precisamos estender o conceito de grau de Brouwer para funções cujos domínios são o fecho de subconjuntos abertos e limitados de um espaço de Banach qualquer. Para isso, vamos apresentar a teoria de grau de Leray-Schauder. As propriedades para grau de Brouwer podem ser estendidas para grau de Leray-Schauder (veja [9]). Entretanto, enunciaremos aqui apenas as propriedades utilizadas neste trabalho.

Definição 3.13. Sejam $X$ um espaço de Banach e $U \subset X$ um conjunto aberto e limitado. Considere $f: \bar{U} \rightarrow X$ uma função compacta tal que $z \notin(I-f)(\partial U)$, onde I denota a função identidade. O grau de Leray-Schauder é uma função denotada por deg $g_{L S}$, que associa a cada tripla $[I-f, U, z]$ um número $\operatorname{deg}_{L S}[I-f, U, z] \in \mathbb{Z}$, onde $\mathbb{Z}$ denota o conjunto dos números inteiros, e que satisfaz as seguintes propriedades:

(i) $\operatorname{deg}_{L S}[I, U, y]=1$, para $y \in U$.

(ii) Se $\operatorname{deg}_{L S}[I-f, U, z] \neq 0$, então $z \in(I-f)(U)$.

(iii) $\operatorname{deg}_{L S}(I-H(t, \cdot), U, y(t))$ independe de $t \in[0,1]$, sempre que $H:[0,1] \times \bar{U} \rightarrow X$ for uma função compacta e $y:[0,1] \rightarrow X$ for uma função contínua tal que

$$
y(t) \notin(I-H(t, \cdot))(\partial U) e m[0,1] .
$$

A seguir, temos uma proposição que caracteriza a função grau de Leray-Schauder para o caso particular em que a imagem da função $f$ da Definição 3.13 está contida num subespaço de dimensão finita de $X$ e o resultado pode ser encontrado em [4]. 
Proposição 3.14. Sejam $X$ um espaço de Banach, $U \subset X$ um aberto limitado e $f \in C(\bar{U}, X)$ uma aplicação cuja imagem está contida em algum subespaço $F$ de $X$ de dimensão finita. Definimos o grau de Leray-Schauder de $I-f$ em relação a $U$, num ponto $b \notin(I-f)(\partial U)$, como sendo o número inteiro

$$
\operatorname{deg}_{L S}(I-f, U, b)=\operatorname{deg}\left(\left.(I-f)\right|_{\bar{U} \cap F}, U \cap F, b\right),
$$

sendo que o lado direito direito da igualdade acima é a função grau de Brouwer dada pelo Teorema 2.8, presente no Capítulo 2.

Agora, vamos definir o conceito de grau de Leray-Schauder local o qual iremos chamar de indexação de Leray-Schauder.

Definição 3.15. Considere $f: \bar{U} \rightarrow X$ nas condições da Definição 3.13. Se a for um zero isolado de $f$ e $B(a, r)$ denotar a bola aberta centrada em a e de raio $r>0$, então a função que associa cada tripla $[I-f, B(a, r), 0]$ um número inteiro $\operatorname{deg}_{L S}[I-f, B(a, r), 0]$ estará bem definida e independerá da escolha de $r$, para $r>0$ suficientemente pequeno. Este valor será chamado de indexação local de Leray-Schauder de I- f em a e denotamos tal valor por $\operatorname{ind}_{L S}[I-f, a]$.

O resultado seguinte pode ser encontrado em [6] e diz que a EDOG (3.17) possui pelo menos uma solução $T$-periódica, sob certas condições.

Teorema 3.16. Seja $F \in \mathcal{F}\left(\Omega, h_{R}, \omega_{R}\right)$, onde $\Omega=B_{R} \times[0, T]$, e suponha que exista um conjunto aberto e limitado $\Delta \subset B V$ tal que as seguintes condições são válidas:

(i) Para cada $\lambda \in(0,1]$, a equação

$$
\frac{d x}{d \tau}=\lambda D F(x, t)
$$

não admite solução T-periódica $x$ em $B V$ tal que $x \in \partial \Delta$. 
(ii) A equação

$$
\psi(a):=\int_{0}^{T} D F(a, t)=0
$$

não admite solução $a \in \partial \Delta \cap \mathbb{R}^{n}$ (onde $\mathbb{R}^{n}$ é o conjunto das funções constantes em $B V)$.

(iii) $\operatorname{deg}\left(\psi, \Delta \cap \mathbb{R}^{n}, 0\right) \neq 0$.

Então a EDOG (3.17) tem pelo menos uma solução T-periódica $x \in \Delta$.

Demonstração. Vamos definir o operador $\mathcal{H}: \bar{\Delta} \times[0,1] \rightarrow B V$ da seguinte forma

$$
x(s)=x(0)+\int_{0}^{T} D F(x(\tau), t)+\lambda \int_{0}^{s} D F(x(\tau), t)=: \mathcal{H}(x, \lambda)(s)
$$

parametrizado por $\lambda \in[0,1]$, para todo $s \in[0, T]$. Primeiramente, tomemos $\lambda=1$. Pelo Teorema 3.11, os pontos fixos do operador $\mathcal{H}$ são as soluções $T$-periódicas de (3.17). Portanto, pela hipótese (i), obtemos $\mathcal{H}(x, 1) \neq x$ para todo $x \in \partial \Delta$.

Para $\lambda \in(0,1)$, se $x$ for um ponto fixo do operador $\mathcal{H}$, então tomando $s=0$ em (3.20), obtemos

$$
\int_{0}^{T} D F(x(\tau), t)=0
$$

e tomando $s=T$ em (3.20), temos

$$
x(T)=x(0)+\int_{0}^{T} D F(x(\tau), t)+\lambda \int_{0}^{T} D F(x(\tau), t) .
$$

Portanto,

$$
x(T)=x(0)+\lambda \int_{0}^{T} D F(x(\tau), t)
$$

Então $x$ é uma solução de (3.18) e, por $(3.21), x(T)=x(0)$. Portanto $x$ é uma solução $T$-periódica de (3.18). Logo, para cada $\lambda \in(0,1)$, os pontos fixos de $\mathcal{H}(\cdot, \lambda)$ são soluções $T$-periódicas de (3.18). Assim, pela hipótese (i), $\mathcal{H}(x, \lambda) \neq x$ para $\lambda \in(0,1)$ e $x \in \partial \Delta$. 
Agora, consideremos o caso em que $\lambda=0$. A equação (3.20) pode ser escrita como

$$
x(s)=x(0)+\int_{0}^{T} D F(x(\tau), t), \quad s \in[0, T],
$$

o que implica que $x$ é constante, ou seja, $x(s)=a$ em $[0, T]$. Portanto, usando (3.22), obtemos

$$
\psi(a)=\int_{0}^{T} D F(a, t)=0 .
$$

Pela hipótese (ii), $\mathcal{H}(x, 0) \neq x$ para todo $x \in \partial \Delta$. Portanto, combinando os casos acima, podemos concluir que se

$$
\mathcal{H}(u, \lambda) \neq u, \quad \text { para qualquer par }(u, \lambda) \in \partial \Delta \times[0,1]
$$

então teremos

$$
0 \notin I-\mathcal{H}(\cdot, \lambda)(\partial \Delta), \lambda \in[0,1]
$$

Daí, usando as propriedades de invariância homotópica de grau de Leray-Schauder presente na Definição 3.13, obtemos

$$
\operatorname{deg}_{L S}[I-\mathcal{H}(\cdot, 1), \Delta, 0]=\operatorname{deg}_{L S}[I-\mathcal{H}(\cdot, 0), \Delta, 0]
$$

Como $\mathcal{H}(\bar{\Delta},\{0\}) \subset \mathbb{R}^{n}$ e usando a Definição 3.14 de grau de Leray-Schauder e a Observação 2.10 do Capítulo 2, obtemos

$$
\begin{gathered}
\operatorname{deg}_{L S}[I-\mathcal{H}(\cdot, 0), \Delta, 0]=\operatorname{deg}\left(\left(I-\left.\mathcal{H}(\cdot, 0)\right|_{\mathbb{R}^{n}}, \Delta \cap \mathbb{R}^{n}, 0\right)=\right. \\
=\operatorname{deg}\left(-\psi, \Delta \cap \mathbb{R}^{n}, 0\right)=(-1)^{n} \operatorname{deg}\left(\psi, \Delta \cap \mathbb{R}^{n}, 0\right) \neq 0 .
\end{gathered}
$$

Logo a equação (3.24) é diferente de zero pela hipótese (iii). Portanto

$$
d_{L S}[I-\mathcal{H}(\cdot, 1), \Delta, 0] \neq 0
$$


Assim, pelo item (ii) da Definição 3.13, obtemos

$$
0 \in I-\mathcal{H}(\cdot, 1)(\Delta)
$$

o que implica que existe $x \in \Delta$ tal que $I(x)-\mathcal{H}(x, 1)=0$, ou seja,

$$
\mathcal{H}(x, 1)=x .
$$

Logo $x \in \Delta$ é um ponto fixo de $\mathcal{H}(\cdot, 1)$ e, consequentemente, pelo Teorema $3.11, x$ é uma solução $T$-periódica de (3.17).

\subsection{Teoria de bifurcação para EDOGs}

Nesta seção, nosso objetivo é definir ponto de bifurcação de uma solução $T$-periódica de uma EDOG do tipo

$$
\frac{d x}{d \tau}=D F_{t}(\lambda, x, t)
$$

e provar um resultado sobre a existência de ponto de bifurcação para soluções periódicas de (3.25), utilizando um operador similar a $\mathcal{M}$, como definido em (3.9), o qual definiremos ao longo desta seção. Para isso, vamos considerar $T>0, \widetilde{\Omega}=[0,1] \times B_{R} \times[0, T]$, onde $B_{R} \subset \mathbb{R}^{n}$ é a bola aberta centrada na origem e de raio $R>0$. Também vamos considerar $h_{R}:[0, T] \rightarrow \mathbb{R}$ uma função não decrescente e contínua à esquerda, $\omega_{R}:[0,+\infty) \rightarrow \mathbb{R}$ uma função contínua, crescente e que satisfaz $\omega_{R}(0)=0$.

Denotaremos por $\widetilde{\mathcal{F}}\left(\widetilde{\Omega}, h_{R}, \omega_{R}\right)$ a classe das funções $F:[0,1] \times B_{R} \times[0, T] \rightarrow \mathbb{R}^{n}$ tais que as seguintes desigualdades são satisfeitas:

$$
\begin{gathered}
\left\|F\left(\lambda, z, t_{2}\right)-F\left(\lambda, z, t_{1}\right)\right\| \leq\left|h_{R}\left(t_{2}\right)-h_{R}\left(t_{1}\right)\right| \\
\left\|F\left(\lambda, z, t_{2}\right)-F\left(\lambda, z, t_{1}\right)-F\left(\lambda, y, t_{2}\right)+F\left(\lambda, y, t_{1}\right)\right\| \leq \omega_{R}(\|z-y\|)\left|h_{R}\left(t_{2}\right)-h_{R}\left(t_{1}\right)\right|,
\end{gathered}
$$


para quaisquer $\lambda \in[0,1], z, y \in B_{R}$ e $t_{1}, t_{2} \in[0, T]$.

Neste caso, vamos considerar a EDOG

$$
\frac{d x}{d \tau}=D_{t} F(\lambda, x, t)
$$

como na Definição 3.1, onde $F \in \widetilde{\mathcal{F}}\left(\widetilde{\Omega}, h_{R}, \omega_{R}\right)$ e vamos considerar, também, o conjunto $B V^{*}$ definido por

$$
B V^{*}=\left\{x:[0,1] \times[0, T] \rightarrow \mathbb{R}^{n} ; \text { para cada } \lambda \in[0,1], x(\lambda, \cdot) \text { pertence a } B V\right\}
$$

e vamos definir o operador

$$
\begin{aligned}
\Phi:[0,1] \times B V^{*} & \rightarrow B V \\
(\lambda, x) & \mapsto \Phi(\lambda, x)
\end{aligned}
$$

tal que, para cada $s \in[0, T]$,

$$
\Phi(\lambda, x)(s)=x(\lambda, s)-x(\lambda, 0)-\int_{0}^{T} D F(\lambda, x(\lambda, \tau), t)-\int_{0}^{s} D F(\lambda, x(\lambda, \tau), t) .
$$

Segue do Teorema 3.7 (com algumas adaptações) o fato do operador $\Phi$ estar bem definido.

O resultado a seguir pode ser encontrado em [6] e nos diz que existe uma correspondência entre as soluções periódicas de (3.25) e os zeros do operador $\Phi$ definido em (3.28).

Teorema 3.17. Uma função $x:[0,1] \times[0, T] \rightarrow \mathbb{R}^{n}$ será uma solução T-periódica de (3.25), se e somente se, $x$ for um zero do operador $\Phi(\lambda, \cdot)$, onde $\Phi$ é dado por (3.28), ou seja, $\Phi(\lambda, x)(s)=0$, para $s \in[0, T], \lambda \in[0,1]$.

Demonstração. Suponha que $x$ seja uma solução $T$-periódica de (3.25). Isso significa que $x$ é de variação limitada (pelo Teorema 3.6) e que, para todo $s \in[0, T]$ e todo $\lambda \in[0,1]$, vale

$$
x(\lambda, s)=x(\lambda, 0)+\int_{0}^{s} D F(\lambda, x(\lambda, \tau), t) .
$$


Em particular, para todo $\lambda \in[0,1]$

$$
x(\lambda, T)=x(\lambda, 0)+\int_{0}^{T} D F(\lambda, x(\lambda, \tau), t) .
$$

Como por hipótese, para cada $\lambda \in[0,1], x(\lambda, T)=x(\lambda, 0)$, obtemos

$$
\int_{0}^{T} D F(\lambda, x(\lambda, \tau), t)=0
$$

Logo, para todo $s \in[0, T]$ e todo $\lambda \in[0,1]$, temos

$$
x(\lambda, s)=x(\lambda, 0)+\int_{0}^{T} D F(\lambda, x(\lambda, \tau), t)+\int_{0}^{s} D F(\lambda, x(\lambda, \tau), t)
$$

o que implica que

$$
\Phi(\lambda, x)(s)=0, \quad s \in[0, T], \lambda \in[0,1] .
$$

Reciprocamente, suponha que $x \in B V^{*}$ seja um zero de $\Phi(\lambda, \cdot)(s)=0$, para todo $s \in$ $[0, T]$. Tomando $s=0$ em (3.28), obtemos

$$
\int_{0}^{T} D F(\lambda, x(\lambda, \tau), t)=0
$$

Então, pela definição do operador $\Phi$, obtemos

$$
x(\lambda, s)-x(\lambda, 0)=\int_{0}^{s} D F(\lambda, x(\lambda, \tau), t)
$$

Por outro lado, tomando $s=T$ em (3.28) e utilizando (3.31), obtemos para cada $\lambda \in[0,1]$, $x(\lambda, T)=x(\lambda, 0)$. Logo $x$ é uma solução $T$-periódica de (3.25).

Do que vimos acima, encontrar pontos de bifurcação de soluções periódicas de (3.25) é equivalente a encontrar pontos de bifurcação da equação

$$
\Phi(\lambda, x)=0,
$$


onde $\Phi$ é dado por (3.28).

Considere, novamente, o operador $\Phi$ como definido em (3.28). Para o próximo resultado vamos utilizar o fato de que $\Phi(\lambda, 0)=0$, para todo $\lambda \in[0,1]$, ou seja, precisaremos considerar que a solução nula seja solução da EDOG (3.25). Para tanto, assumiremos a seguinte condição:

$$
F(\lambda, 0, t)-F(\lambda, 0, s)=0 \text {, para quaisquer } t, s \in[0, T] \text { e } \lambda \in[0,1] .
$$

Então 0 será uma solução de (3.25) e, portanto,

$$
\Phi(\lambda, 0)(s)=-\int_{0}^{T} D F(\lambda, 0(\lambda, \tau), t)-\int_{0}^{s} D F(\lambda, 0(\lambda, \tau), t)=0,
$$

para todo $\lambda \in[0,1]$ e todo $s \in[0, T]$.

A seguir, vamos apresentar uma definição de ponto de bifurcação da equação $\Phi(\lambda, x)=0$.

Definição 3.18. Um ponto $\left(\lambda_{0}, 0\right)$ será chamado de ponto de bifurcação (ou $\lambda_{0}$ será um ponto de bifurcação com respeito à solução trivial) da equação

$$
\Phi(\lambda, x)=0
$$

se toda vizinhança de $\left(\lambda_{0}, 0\right)$ em $[0,1] \times B V^{*}$ contiver uma solução $(\lambda, x)$ de $\Phi(\lambda, x)=0$ tal que $x \neq 0$.

Ainda para o próximo resultado, vamos considerar as seguintes notações sobre o operador $\Phi$, como definido em (3.28). Considere o operador identidade

$$
\begin{aligned}
I: & {[0,1] \times B V^{*} \rightarrow B V } \\
(\lambda, x) & \mapsto \quad I(\lambda, x)=x(\lambda, \cdot)
\end{aligned}
$$

onde

$$
x(\lambda, \cdot):[0, T] \rightarrow \mathbb{R}^{n}
$$




$$
t \mapsto x(\lambda, t)
$$

e o operador $G$ como definido abaixo

$$
G:[0,1] \times B V^{*} \rightarrow B V
$$

$$
(\lambda, x) \quad \mapsto \quad G(\lambda, x)
$$

tal que, para cada $t \in[0, T]$,

$$
G(\lambda, x)(t)=x(\lambda, 0)+\int_{0}^{T} D F(\lambda, x(\lambda, \tau), s)+\int_{0}^{t} D F(\lambda, x(\lambda, \tau), s) .
$$

Observemos que o operador $\Phi$, definido em (3.28), pode ser escrito da seguinte forma

$$
\Phi(\lambda, x)(t)=I(\lambda, x)(t)-G(\lambda, x)(t)
$$

para todo $t \in[0, T]$ e todo $\lambda \in[0,1]$.

Proposição 3.19. Seja $F \in \widetilde{\mathcal{F}}\left(\widetilde{\Omega}, h_{R}, \omega_{R}\right)$. Então, para cada $\lambda \in[0,1]$ fixado, o operador $G(\lambda, \cdot): B V \rightarrow B V$ descrito por (3.33) será compacto.

Demonstração. Basta mostrarmos que, se $\left\{x_{n}\right\}_{n \in \mathbb{N}}$ for uma sequência limitada em $B V$, então para cada $\lambda \in[0,1]$ fixado, $\left\{G\left(\lambda, x_{n}\right)\right\}_{n \in \mathbb{N}}$ possuirá uma subsequência convergente em $B V$.

Suponha que $\left\{x_{n}\right\}_{n \in \mathbb{N}}$ seja uma sequência limitada em $B V$. Pelo Teorema da Escolha de Helly (veja [11]), existe uma subsequência $\left\{x_{n_{k}}\right\}_{k \in \mathbb{N}}$ de $\left\{x_{n}\right\}_{n \in \mathbb{N}}$, a qual converge pontualmente em $[0, T]$ para uma função $y \in B V$.

Por simplicidade, podemos denotar a subsequência acima por $\left\{x_{n}\right\}_{n \in \mathbb{N}}$. Logo, para cada $\lambda$ fixo, $\left\{x_{n}(\lambda, t)\right\}_{n \in \mathbb{N}}$ converge pontualmente para $y(\lambda, t)$ em $[0, T]$. Assim,

$$
\begin{gathered}
\left\|G\left(\lambda, x_{n}\right)-G(\lambda, y)\right\|_{B V}=\left\|G\left(\lambda, x_{n}\right)(0)-G(\lambda, y)(0)\right\|+0^{T}\left[G\left(\lambda, x_{n}\right)-G(\lambda, y)\right] \\
\leq\left\|x_{n}(\lambda, 0)-y(\lambda, 0)\right\|+\left\|\int_{0}^{T} D\left[F\left(\lambda, x_{n}(\lambda, \tau), t\right)-F(\lambda, y(\lambda, \tau), t)\right]\right\|+_{0}^{T}\left[G\left(\lambda, x_{n}\right)-G(\lambda, y)\right] .
\end{gathered}
$$


Através de uma adaptação do Lema 3.8, para cada $\lambda \in[0,1]$ fixado, pode-se obter

$\left\|\int_{0}^{T} D\left[F\left(\lambda, x_{n}(\lambda, \tau), t\right)-F(\lambda, y(\lambda, \tau), t)\right]\right\| \leq \int_{0}^{T} \omega_{R}\left(\left\|x_{n}(\lambda, t)-y(\lambda, t)\right\|\right) d h_{R}(t)$.

Notemos que, para cada $\lambda \in[0,1]$ fixado e para cada $0 \leq t_{i-i} \leq t_{i} \leq T$, onde $d=$ $\left(\left[t_{i-1}, t_{i}\right]\right)_{i=1}^{|d|}$ é uma divisão de $[0, T]$ e utilizando (3.34), temos

$$
\begin{gathered}
\sum_{i=1}^{|d|}\left\|G\left(\lambda, x_{n}\right)\left(t_{i}\right)-G(\lambda, y)\left(t_{i}\right)-G\left(\lambda, x_{n}\right)\left(t_{i-1}\right)+G(\lambda, y)\left(t_{i-1}\right)\right\|= \\
\sum_{i=1}^{|d|}\left\|\int_{t_{i-1}}^{t_{i}} D\left[F\left(\lambda, x_{n}(\lambda, \tau), t\right)-F(\lambda, y(\lambda, \tau), t)\right]\right\| \\
\leq \sum_{i=1}^{|d|} \int_{t_{i-1}}^{t_{i}} \omega_{R}\left(\left\|x_{n}(\lambda, t)-y(\lambda, t)\right\|\right) d h_{R}(t)=\int_{0}^{T} \omega_{R}\left(\left\|x_{n}(\lambda, t)-y(\lambda, t)\right\|\right) d h_{R}(t) .
\end{gathered}
$$

Tomando o supremo sobre todas as divisões de $[0, T]$ em (3.35), obtemos

$$
{ }_{0}^{T}\left[G\left(\lambda, x_{n}\right)-G(\lambda, y)\right] \leq \int_{0}^{T} \omega_{R}\left(\left\|x_{n}(\lambda, t)-y(\lambda, t)\right\|\right) d h_{R}(t)
$$

Portanto,

$$
\begin{gathered}
\left\|G\left(\lambda, x_{n}\right)-G(\lambda, y)\right\|_{B V} \leq\left\|G\left(\lambda, x_{n}\right)(0)-G(\lambda, y)(0)\right\|+_{0}^{T}\left[G\left(\lambda, x_{n}\right)-G(\lambda, y)\right] \\
\leq\left\|x_{n}(\lambda, 0)-y(\lambda, 0)\right\|+2 \int_{0}^{T} \omega_{R}\left(\left\|x_{n}(\lambda, t)-y(\lambda, t)\right\|\right) d h_{R}(t)
\end{gathered}
$$

Daí, fazendo $n$ tender a infinito em (3.36) e observando que, para cada $\lambda \in[0,1]$ fixado, a sequência $\left\{x_{n}(\lambda, t)\right\}_{n \in \mathbb{N}}$ converge pontualmente para $y(\lambda, t)$ em $[0, T]$, então o lado direito de (3.36) tende a zero.

O próximo resultado pode ser encontrado em [6] e nos mostra uma importante propriedade que será utilizada mais à frente. 
Proposição 3.20. Sejam $F:[0,1] \times B_{R} \times[0, T] \rightarrow \mathbb{R}^{n}$ e $x:[0,1] \times[0, T] \rightarrow B_{R}$ uma função de variação limitada e suponha que exista uma função $h:[0, T] \rightarrow \mathbb{R}$ não decrescente, contínua à esquerda, tal que

$$
\begin{aligned}
\| F\left(\lambda_{1}, x\left(\lambda_{1}, \tau\right), t\right)+ & F\left(\lambda_{2}, x\left(\lambda_{2}, \tau\right), s\right)-F\left(\lambda_{2}, x\left(\lambda_{2}, \tau\right), t\right)-F\left(\lambda_{1}, x\left(\lambda_{1}, \tau\right), s\right) \| \\
& \leq\left\|x\left(\lambda_{1}, \tau\right)-x\left(\lambda_{2}, \tau\right)\right\||h(t)-h(s)|
\end{aligned}
$$

para quaisquer $\lambda_{1}, \lambda_{2} \in[0,1], s, t \in[0, T]$ e $\tau \in[s, t]$. Então, para $\lambda_{1}, \lambda_{2} \in[0,1]$, temos

$$
\| \int_{0}^{T} D\left[F\left(\lambda_{1}, x\left(\lambda_{1}, \tau\right), t\right)-F\left(\lambda_{2}, x\left(\lambda_{2}, \tau\right), t\right)\left\|\leq \int_{0}^{T}\right\| x\left(\lambda_{1}, t\right)-x\left(\lambda_{2}, t\right) \| d h(t) .\right.
$$

Demonstração. Para uma divisão marcada $d=\left(\tau_{i},\left[s_{i-1}, s_{i}\right]\right)_{i=1}^{|d|}$ arbitrária de $[0, T]$, temos

$$
\begin{gathered}
\left\|\sum_{i=1}^{|d|} F\left(\lambda_{1}, x\left(\lambda_{1}, \tau_{i}\right), s_{i}\right)-F\left(\lambda_{2}, x\left(\lambda_{2}, \tau_{i}\right), s_{i}\right)-F\left(\lambda_{1}, x\left(\lambda_{1}, \tau_{i}\right), s_{i-1}\right)+F\left(\lambda_{2}, x\left(\lambda_{2}, \tau_{i}\right), s_{i-1}\right)\right\| \\
\leq \sum_{i=1}^{|d|}\left\|F\left(\lambda_{1}, x\left(\lambda_{1}, \tau_{i}\right), s_{i}\right)-F\left(\lambda_{2}, x\left(\lambda_{2}, \tau_{i}\right), s_{i}\right)-F\left(\lambda_{1}, x\left(\lambda_{1}, \tau_{i}\right), s_{i-1}\right)+F\left(\lambda_{2}, x\left(\lambda_{2}, \tau_{i}\right), s_{i-1}\right)\right\| \\
\leq \sum_{i=1}^{|d|}\left\|x\left(\lambda_{1}, \tau_{i}\right)-x\left(\lambda_{2}, \tau_{i}\right)\right\|\left|h\left(s_{i}\right)-h\left(s_{i-1}\right)\right| .
\end{gathered}
$$

Dado $\epsilon>0$, existe uma divisão marcada $\delta$-fina $d=\left(\tau_{i},\left[s_{i-1}, s_{i}\right]\right)_{i=1}^{|d|}$ de $[0, T]$ tal que

$$
\begin{aligned}
& \| \int_{0}^{T} D\left[F\left(\lambda_{1}, x\left(\lambda_{1}, \tau\right), t\right)-F\left(\lambda_{2}, x\left(\lambda_{2}, \tau\right), t\right)\right]-\sum_{i=1}^{|d|}\left[F\left(\lambda_{1}, x\left(\lambda_{1}, \tau_{i}\right), s_{i}\right)\right. \\
& \left.-F\left(\lambda_{2}, x\left(\lambda_{2}, \tau_{i}\right), s_{i}\right)-F\left(\lambda_{1}, x\left(\lambda_{1}, \tau_{i}\right), s_{i-1}\right)+F\left(\lambda_{2}, x\left(\lambda_{2}, \tau_{i}\right), s_{i-1}\right)\right] \|<\epsilon
\end{aligned}
$$

e

$$
\left|\int_{0}^{T}\left\|x\left(\lambda_{1}, t\right)-x\left(\lambda_{2}, t\right)\right\| d h(t)-\sum_{i=1}^{|d|}\left\|x\left(\lambda_{1}, \tau_{i}\right)-x\left(\lambda_{2}, \tau_{i}\right)\right\|\left[h\left(s_{i}\right)-h\left(s_{i-1}\right)\right]\right|<\epsilon .
$$


Logo, obtemos

$$
\left\|\int_{0}^{T} D\left[F\left(\lambda_{1}, x\left(\lambda_{1}, \tau\right), t\right)-F\left(\lambda_{2}, x\left(\lambda_{2}, \tau\right), t\right)\right]\right\| \leq
$$

$$
\begin{gathered}
\left\|\sum_{i=1}^{|d|}\left[F\left(\lambda_{1}, x\left(\lambda_{1}, \tau_{i}\right), s_{i}\right)-F\left(\lambda_{2}, x\left(\lambda_{2}, \tau_{i}\right), s_{i}\right)-F\left(\lambda_{1}, x\left(\lambda_{1}, \tau_{i}\right), s_{i-1}\right)+F\left(\lambda_{2}, x\left(\lambda_{2}, \tau_{i}\right), s_{i-1}\right)\right]\right\| \\
+\| \int_{0}^{T} D\left[F\left(\lambda_{1}, x\left(\lambda_{1}, \tau\right), t\right)-F\left(\lambda_{2}, x\left(\lambda_{2}, \tau\right), t\right)-\right.
\end{gathered}
$$

$$
\begin{gathered}
\sum_{i=1}^{|d|}\left[F\left(\lambda_{1}, x\left(\lambda_{1}, \tau_{i}\right), s_{i}\right)-F\left(\lambda_{2}, x\left(\lambda_{2}, \tau_{i}\right), s_{i}\right)-F\left(\lambda_{1}, x\left(\lambda_{1}, \tau_{i}\right), s_{i-1}\right)+F\left(\lambda_{2}, x\left(\lambda_{2}, \tau_{i}\right), s_{i-1}\right)\right] \| \\
<\sum_{i=1}^{|d|}\left\|x\left(\lambda_{1}, \tau_{i}\right)-x\left(\lambda_{2}, \tau_{i}\right)\right\|\left(h\left(s_{i}\right)-h\left(s_{i-1}\right)\right)+\epsilon \\
\leq\left|\sum_{i=1}^{|d|}\left\|x\left(\lambda_{1}, \tau_{i}\right)-x\left(\lambda_{2}, \tau_{i}\right)\right\|\left(h\left(s_{i}\right)-h\left(s_{i-1}\right)\right)-\int_{0}^{T}\left\|x\left(\lambda_{1}, t\right)-x\left(\lambda_{2}, t\right)\right\| d h(t)\right| \\
+\int_{0}^{T}\left\|x\left(\lambda_{1}, t\right)-x\left(\lambda_{2}, t\right)\right\| d h(t)+\epsilon \\
<2 \epsilon+\int_{0}^{T}\left\|x\left(\lambda_{1}, t\right)-x\left(\lambda_{2}, t\right)\right\| d h(t) .
\end{gathered}
$$

Daí, fazendo $\epsilon$ tender a zero, obtemos o resultado.

O teorema a seguir pode ser encontrado em [6] e nos diz que, para cada $x \in B V^{*}$ fixado, o operador $G(\cdot, x):[0,1] \rightarrow B V$, dado por $(3.33)$, será uniformemente contínuo com relação à primeira variável.

Teorema 3.21. Suponha satisfeitas as condições da Proposição 3.20 e que $x:[0,1] \times[0, T] \rightarrow$ $\mathbb{R}^{n}$ seja lipschitziana na primeira variável, com constante de Lipschitz $L>0$. Então, para cada $x \in B V^{*}$ fixado, $G(\cdot, x):[0,1] \rightarrow B V$, dado por (3.33), será uniformemente contínuo.

Demonstração. Precisamos mostrar que, dado $\epsilon>0$, existe $\bar{\delta}>0$ tal que se $\left|\lambda_{1}-\lambda_{2}\right|<\bar{\delta}$, então

$$
\left\|G\left(\lambda_{1}, x\right)-G\left(\lambda_{2}, x\right)\right\|_{B V}<\epsilon
$$


para quaisquer $\lambda_{1}, \lambda_{2} \in[0,1]$. De fato, temos

$$
\begin{aligned}
\left\|G\left(\lambda_{1}, x\right)-G\left(\lambda_{2}, x\right)\right\|_{B V}= & \left\|G\left(\lambda_{1}, x\right)(0)-G\left(\lambda_{2}, x\right)(0)\right\|+_{0}^{T}\left[G\left(\lambda_{1}, x\right)-G\left(\lambda_{2}, x\right)\right] \\
\leq\left\|x\left(\lambda_{1}, 0\right)-x\left(\lambda_{2}, 0\right)\right\|+ & \left\|\int_{0}^{T} D\left[F\left(\lambda_{1}, x\left(\lambda_{1}, \tau\right), s\right)-F\left(\lambda_{2}, x\left(\lambda_{2}, \tau\right), s\right)\right]\right\| \\
& +{ }_{0}^{T}\left[G\left(\lambda_{1}, x\right)-G\left(\lambda_{2}, x\right)\right] .
\end{aligned}
$$

Seja uma divisão arbitrária $d=\left(\left[s_{i-1}, s_{i}\right]\right)_{i=1}^{|d|}$ de $[0, T]$. Então

$$
\begin{gathered}
\sum_{i=1}^{|d|}\left\|G\left(\lambda_{1}, x\right)\left(t_{i}\right)-G\left(\lambda_{2}, x\right)\left(t_{i}\right)-G\left(\lambda_{1}, x\right)\left(t_{i-1}\right)+G\left(\lambda_{2}, x\right)\left(t_{i-1}\right)\right\|= \\
=\sum_{i=1}^{|d|} \| x\left(\lambda_{1}, 0\right)+\int_{0}^{T} D F\left(\lambda_{1}, x\left(\lambda_{1}, \tau\right), s\right)+\int_{0}^{t_{i}} D F\left(\lambda_{1}, x\left(\lambda_{1}, \tau\right), s\right) \\
-x\left(\lambda_{2}, 0\right)-\int_{0}^{T} D F\left(\lambda_{2}, x\left(\lambda_{2}, \tau\right), s\right)-\int_{0}^{t_{i}} D F\left(\lambda_{2}, x\left(\lambda_{2}, \tau\right), s\right) \\
-x\left(\lambda_{1}, 0\right)-\int_{0}^{T} D F\left(\lambda_{1}, x\left(\lambda_{1}, \tau\right), s\right)-\int_{0}^{t_{i-1}} D F\left(\lambda_{1}, x\left(\lambda_{1}, \tau\right), s\right) \\
+x\left(\lambda_{2}, 0\right)+\int_{0}^{T} D F\left(\lambda_{2}, x\left(\lambda_{2}, \tau\right), s\right)+\int_{0}^{t_{i-1}} D F\left(\lambda_{2}, x\left(\lambda_{2}, \tau\right), s\right) \| \\
=\sum_{i=1}^{|d|}\left\|\int_{t_{i-1}}^{t_{i}} D\left[F\left(\lambda_{1}, x\left(\lambda_{1}, \tau\right), s\right)-F\left(\lambda_{2}, x\left(\lambda_{2}, \tau\right), s\right)\right]\right\| \\
\leq \sum_{i=1}^{|d|} \int_{t_{i-1}}^{t_{i}}\left\|x\left(\lambda_{1}, s\right)-x\left(\lambda_{2}, s\right)\right\| d h(s)=\int_{0}^{T}\left\|x\left(\lambda_{1}, s\right)-x\left(\lambda_{2}, s\right)\right\| d h(s),
\end{gathered}
$$

onde utilizamos a Proposição 3.20.

Tomando o supremo sobre todas as divisões $d=\left[s_{i-1}, s_{i}\right]_{i=1}^{|d|}$ de $[0, T]$, obtemos

$$
{ }_{0}^{T}\left[G\left(\lambda_{1}, x\right)-G\left(\lambda_{2}, x\right)\right] \leq \int_{0}^{T}\left\|x\left(\lambda_{1}, s\right)-x\left(\lambda_{2}, s\right)\right\| d h(s .)
$$


Portanto, temos

$$
\begin{gathered}
\left\|G\left(\lambda_{1}, x\right)-G\left(\lambda_{2}, x\right)\right\|_{B V} \leq\left\|x\left(\lambda_{1}, 0\right)-x\left(\lambda_{2}, 0\right)\right\|+2 \int_{0}^{T}\left\|x\left(\lambda_{1}, t\right)-x\left(\lambda_{2}, t\right)\right\| d h(t) \\
\leq L\left|\lambda_{1}-\lambda_{2}\right|+2 \sup _{t \in[0, T]}\left\|x\left(\lambda_{1}, t\right)-x\left(\lambda_{2}, t\right)\right\| \int_{0}^{T} d h(t) \\
\leq L\left|\lambda_{1}-\lambda_{2}\right|+2 \sup _{t \in[0, T]}\left\|x\left(\lambda_{1}, t\right)-x\left(\lambda_{2}, t\right)\right\|(h(T)-h(0)) \\
\leq L\left|\lambda_{1}-\lambda_{2}\right|+2 L\left|\lambda_{1}-\lambda_{2}\right|(h(T)-h(0)),
\end{gathered}
$$

onde usamos o fato de que $x$ é lipschitziana em relação à primeira variável. Logo, para cada $x \in B V^{*}$ fixado, $G(\cdot, x)$ é uniformemente contínua em $[0,1]$.

Agora, estamos aptos a provar o próximo teorema. O próximo resultado pode ser encontrado em [6] e é uma adaptação para as equações diferenciais ordinárias generalizadas do Teorema 26.5, presente em [1].

Teorema 3.22. Seja $\Phi:[0,1] \times B V^{*} \rightarrow B V$, onde $\Phi$ é o operador em (3.28). Suponha que $F \in \widetilde{\mathcal{F}}\left(\widetilde{\Omega}, h_{R}, \omega_{R}\right)$, para todo $R>0$, e que existe $\lambda_{0} \in[0,1]$ tais que as seguintes condições são satisfeitas:

(i) $F(\lambda, 0, t)-F(\lambda, 0, s)=0$, para todo $t, s \in[0, T], \lambda \in[0,1]$.

(ii) Para cada $\lambda \in[0,1] \backslash\left\{\lambda_{0}\right\}=J$, existe uma vizinhança $J_{\lambda} \times U_{\lambda}$ de $\left(\lambda_{0}, 0\right)$ em $J \times B V^{*}$ tal que 0 é o único zero de $G(\mu, \cdot)$ em $U_{\lambda}$, para todo $\mu \in J_{\lambda}$.

(iii) $\left\|F\left(\lambda_{1}, x\left(\lambda_{1}, \tau\right), t\right)+F\left(\lambda_{2}, x\left(\lambda_{2}, \tau\right), s\right)-F\left(\lambda_{2}, x\left(\lambda_{2}, \tau\right), t\right)-F\left(\lambda_{1}, x\left(\lambda_{1}, \tau\right), s\right)\right\|$

$$
\leq\left\|x\left(\lambda_{1}, \tau\right)-x\left(\lambda_{2}, \tau\right)\right\|\left|h_{R}(t)-h_{R}(s)\right|,
$$

para quaisquer $\lambda_{1}, \lambda_{2} \in[0,1], s, t \in[0, T]$ e $\tau \in[s, t]$. 
Então $\left(\lambda_{0}, 0\right)$ será um ponto de bifurcação da equação $\Phi(\lambda, x)=0$, se a indexação local, denotada por $\operatorname{ind}_{L S}[I-G(\lambda, \cdot), 0]$, satisfizer

$$
\operatorname{ind}_{L S}[I-G(0, \cdot), 0] \neq \operatorname{ind}_{L S}[I-G(1, \cdot), 0] .
$$

Demonstração. Façamos a prova por contradição. Suponha que $\left(\lambda_{0}, 0\right)$ não seja um ponto de bifurcação de $\Phi(\lambda, x)=0$. Então, para todo $\lambda \in[0,1]$, existe uma vizinhança $J_{\lambda} \times U_{\lambda}$ de $\left(\lambda_{0}, 0\right) \in[0,1] \times B V^{*}$, satisfazendo

$$
\Phi(\lambda, x) \neq 0, \text { para todo }(\lambda, x) \in J_{\lambda} \times\left(\bar{U}_{\lambda} \backslash\{0\}\right) .
$$

Pela compacidade de $[0,1]$, podemos encontrar um número finito de pontos $\lambda_{1}, \ldots, \lambda_{m} \in$ $[0,1]$ tais que $J_{\lambda_{1}}, \ldots, J_{\lambda_{m}}$ é uma cobertura do intervalo $[0,1]$.

Seja

$$
U=\bigcap_{j=1}^{m} U_{\lambda_{j}} .
$$

Então $\Phi(\lambda, x) \neq 0$, para todo $\operatorname{par}(\lambda, x) \in[0,1] \times \bar{U} \backslash\{0\}$, o que implica que

$$
G(\lambda, x) \neq I(\lambda, x) \text {, para todo par }(\lambda, x) \in[0,1] \times \partial U,
$$

ou seja,

$$
0 \notin(I-G(\lambda, \cdot))(\partial U), \text { para todo } \lambda \in[0,1] \text {. }
$$

Consequentemente, pela propriedade da homotopia de grau de Leray-Schauder (veja (iii) da Definição 3.13), obtemos

$$
\operatorname{deg}_{L S}[I-G(0, \cdot), U, 0]=\operatorname{deg}_{L S}[I-G(1, \cdot), U, 0] .
$$

Portanto,

$$
\operatorname{ind}_{L S}[I-G(0, \cdot), 0]=\operatorname{deg}_{L S}[I-G(0, \cdot), U, 0]=
$$




$$
=\operatorname{deg}_{L S}[I-G(1, \cdot), U, 0]=i n d_{L S}[I-G(1, \cdot), 0]
$$

o que contradiz a hipótese. Logo o teorema está provado.

\subsection{Aplicações}

Nesta seção, nosso objetivo é estudar a correspondência entre as EDOs clássicas e as EDOs generalizadas e posteriormente aplicar os resultados presentes na seção anterior às EDOs clássicas.

Considere a função $f: \mathbb{R}^{n} \times[0, T] \rightarrow \mathbb{R}^{n}$ satisfazendo as seguintes condições:

(i) Para cada $x \in B V$,

$$
f(x(\cdot), \cdot) \text { é Kuzweil-Henstock integrável em }[0, T],
$$

(ii) Existe uma função Lebesgue integrável $M:[0, T] \rightarrow \mathbb{R}$ tal que

$$
\left\|\int_{t_{1}}^{t_{2}} f(x(s), s) d s\right\| \leq \int_{t_{1}}^{t_{2}} M(s) d s
$$

para qualquer $x \in B V$ e quaisquer $t_{1}, t_{2} \in[0, T]$.

(iii) Existe uma função Lebesgue integrável $L:[0, T] \rightarrow \mathbb{R}$ tal que

$$
\left\|\int_{t_{1}}^{t_{2}} f(x(s), s)-f(y(s), s) d s\right\| \leq \int_{t_{1}}^{t_{2}}\|x(s)-y(s)\| L(s) d s
$$

para quaisquer $x, y \in B V$ e quaisquer $t_{1}, t_{2} \in[0, T]$.

Seja $F: \mathbb{R}^{n} \times[0, T] \rightarrow \mathbb{R}^{n}$ definida por

$$
F(z, t)=\int_{0}^{t} f(z, s) d s
$$


para cada $(z, t) \in \mathbb{R}^{n} \times[0, T]$. É fácil verificarmos que $F \in \mathcal{F}(\Omega, h, \omega)$, onde $\Omega=\mathbb{R}^{n} \times[0, T]$,

$$
h(t)=\int_{0}^{t}[M(s)+L(s)] d s, \quad t \in[0, T],
$$

e $\omega:[0,+\infty) \rightarrow \mathbb{R}$ é a função identidade, isto é, $\omega(t)=t$, para $t \in[0,+\infty)$.

Vamos considerar a seguinte equação diferencial ordinária

$$
\dot{x}=f(x, t)
$$

onde $\dot{x}=d x / d t$ e $f: \mathbb{R}^{n} \times[0, T] \rightarrow \mathbb{R}^{n}$ satisfaz (i), (ii) e (iii).

Vamos relembrar o conceito de uma solução de uma equação diferencial ordinária do tipo (3.41). Uma função $x:[0, T] \rightarrow \mathbb{R}^{n}$ será chamada de uma solução de uma EDO do tipo (3.41) em $[0, T]$, se $x$ for diferenciável em $[0, T], x(s) \in \mathbb{R}^{n}$ para todo $s \in[0, T]$ e se para todo $t \in[0, T]$ a equação

$$
\dot{x}=f(x(t), t)
$$

estiver satisfeita.

Integrando a equação (3.42) em relação a $t$, obtemos

$$
x(t)=x(0)+\int_{s_{1}}^{s_{2}} f(x(\tau), \tau) d \tau, \quad t \in[0, T]
$$

onde a integral do lado direito de (3.43) deve ser entendida no sentido de Kurzweil-Henstock e, portanto, pode ser aproximada por uma soma da forma

$$
\sum_{i=1}^{|d|} f\left(x\left(\tau_{i}\right), \tau_{i}\right)\left[t_{i}-t_{i-1}\right]
$$

onde $d=\left(\tau_{i},\left[t_{i-1}, t_{i}\right]\right)_{i=1}^{|d|}$ é uma divisão marcada $\delta$-fina do intervalo $[0, T]$ para uma função calibre $\delta$ adequada. 
Utilizando (3.40), a integral em (3.43) pode ser aproximada por

$$
\sum_{i=1}^{|d|} \int_{t_{i-1}}^{t_{i}} f\left(x\left(\tau_{i}\right), s\right) d s=\sum_{i=1}^{|d|}\left[F\left(x\left(\tau_{i}\right), t_{i}\right)-F\left(x\left(\tau_{i}\right), t_{i-1}\right)\right]
$$

Neste caso, o lado direito de (3.44) aproxima-se da integral não-absoluta de Kurzweil (veja a Definição 3.3) que dá origem à EDOG

$$
\frac{d x}{d \tau}=D F(x, t)
$$

como na Definição 3.4. Portanto toda solução $x:[0, T] \rightarrow \mathbb{R}^{n}$ de (3.45), onde $F$ é dada por (3.40), também é uma solução da EDO (3.41) e vale a recíproca.

Assim, temos o seguinte:

$$
\int_{0}^{s} f(x(t), t) d t=\int_{0}^{s} D F(x(\tau), t), \quad s \in[0, T]
$$

e podemos enunciar o próximo resultado.

Teorema 3.23. Uma função $x:[0, T] \rightarrow \mathbb{R}^{n}$ será uma solução da equação diferencial ordinária (3.41) em $[0, T]$, se e somente se, x for uma solução da equação diferencial ordinária generalizada (3.45) em [0,T], onde a função F é dada em (3.40).

Agora, usando o Teorema 3.23, fica fácil ver que valem os resultados abaixo.

Teorema 3.24. Uma função $x:[0, T] \rightarrow \mathbb{R}^{n}$ de variação limitada será uma solução $T$ periódica de (3.41), se e somente se, $x$ for um ponto fixo do operador $m: B V \rightarrow B V$, onde, para cada $s \in[0, T]$,

$$
m(x)(s)=x(0)+\int_{0}^{T} f(x(t), t) d t+\int_{0}^{s} f(x(t), t) d t .
$$

A seguir, apresentaremos um resultado sobre a existência de soluções periódicas para EDOs, o qual pode ser encontrado em [6]. 
Teorema 3.25. Suponha que exista um subconjunto aberto e limitado $\Delta \subset B V$ tal que as seguintes condições são válidas:

(i) Para cada $\lambda \in(0,1]$, a equação

$$
\dot{x}=\lambda f(x, t)
$$

não admite solução T-periódica $x$ em $B V$ tal que $x \in \partial \Delta$.

(ii) A equação

$$
\phi(a):=\int_{0}^{T} f(a, s) d s=0
$$

não admite solução $a \in \partial \Delta \cap \mathbb{R}^{n}$ (onde $\mathbb{R}^{n}$ é o conjunto das funções constantes em $B V)$.

(iii) $\operatorname{deg}\left(\phi, \Delta \cap \mathbb{R}^{n}, 0\right) \neq 0$.

Então a EDO (3.41) tem pelo menos uma solução T-periódica $x \in \Delta$.

Agora, considere a função $f:[0,1] \times \mathbb{R}^{n} \times[0, T] \rightarrow \mathbb{R}^{n}$ satisfazendo as seguintes condições:

(i) Para cada $x \in B V^{*}$ e cada $\lambda \in[0,1]$,

$$
f(\lambda, x(\lambda, \cdot), \cdot) \text { é Kuzweil-Henstock integrável em }[0, T]
$$

(ii) Existe uma função Lebesgue integrável $M:[0, T] \rightarrow \mathbb{R}$ tal que

$$
\int_{t_{1}}^{t_{2}} f(\lambda, x(\lambda, s), s) d s \leq \int_{t_{1}}^{t_{2}} M(s) d s
$$

para qualquer $x \in B V^{*}$, qualquer $\lambda \in[0,1]$ e quaisquer $t_{1}, t_{2} \in[0, T]$.

(iii) Existe uma função Lebesgue integrável $L:[a, b] \rightarrow \mathbb{R}$ tal que

$$
\left\|\int_{t_{1}}^{t_{2}} f(\lambda, x(\lambda, s), s)-f(\lambda, y(\lambda, s), s) d s\right\| \leq \int_{t_{1}}^{t_{2}}\|x(\lambda, s)-y(\lambda, s)\| L(s) d s
$$

para quaisquer $x, y \in B V^{*}$, qualquer $\lambda \in[0,1]$ e quaisquer $t_{1}, t_{2} \in[0, T]$. 
Seja $F:[0,1] \times \mathbb{R}^{n} \times[0, T] \rightarrow \mathbb{R}^{n}$ definida por

$$
F(\lambda, z, t)=\int_{0}^{t} f(\lambda, z, s) d s
$$

para cada $(\lambda, z, t) \in[0,1] \times \mathbb{R}^{n} \times[0, T]$. É fácil verificarmos que $F \in \mathcal{F}(\Omega, h, \omega)$, onde agora $\Omega=[0,1] \times \mathbb{R}^{n} \times[0, T]$,

$$
h(t)=\int_{0}^{t}[M(s)+L(s)] d s, \quad t \in[0, T]
$$

e $\omega:[0,+\infty) \rightarrow \mathbb{R}$ é a função identidade, isto é, $\omega(t)=t$, para $t \in[0,+\infty)$.

Vamos considerar a seguinte equação diferencial ordinária dependente de parâmetro

$$
\frac{d x}{d t}=f(\lambda, x(\lambda, t), t)
$$

onde $x:[0,1] \times[0, T] \rightarrow \mathbb{R}^{n}$ e $f:[0,1] \times \mathbb{R}^{n} \times[0, T] \rightarrow \mathbb{R}^{n}$ satisfaz (i), (ii) e (iii) acima.

Analogamente ao que fizemos acima, podemos mostrar que vale

$$
\int_{0}^{s} f(\lambda, x(\lambda, t), t) d t=\int_{0}^{s} D F_{t}(\lambda, x(\lambda, \tau), t), \quad s \in[0, T]
$$

onde $x \in B V^{*}$ e $\lambda \in[0,1]$ e assim podemos enunciar o próximo resultado.

Teorema 3.26. Uma função $x:[0,1] \times[0, T] \rightarrow \mathbb{R}^{n}$ será uma solução da equação diferencial ordinária

$$
\frac{d x}{d t}=f(\lambda, x(\lambda, t), t)
$$

em $[0, T]$, se e somente se, $x$ for uma solução da equação diferencial ordinária generalizada

$$
\frac{d x}{d \tau}=D F_{t}(\lambda, x, t)
$$

em $[0, T]$, onde $f:[0,1] \times \mathbb{R}^{n} \times[0, T] \rightarrow \mathbb{R}^{n}$ satisfaz (i), (ii) e (iii) acima e a função $F$ é dada em (3.46). 
Agora, usando o Teorema 3.26, fica fácil ver que vale o resultado abaixo.

Vamos definir o operador

$$
\begin{aligned}
\psi:[0,1] \times B V^{*} & \rightarrow B V \\
(\lambda, x) & \mapsto \psi(\lambda, x)
\end{aligned}
$$

tal que, para cada $s \in[0, T]$,

$$
\psi(\lambda, x)(s)=x(\lambda, s)-x(\lambda, 0)-\int_{0}^{T} f(\lambda, x(\lambda, t), t) d t-\int_{0}^{s} f(\lambda, x(\lambda, t), t) d t .
$$

Vamos considerar também, o operador identidade

$$
\begin{aligned}
& i:[0,1] \times B V^{*} \rightarrow B V \\
& (\lambda, x) \quad \mapsto \quad i(\lambda, x)=x(\lambda, \cdot),
\end{aligned}
$$

e o operador g definido por

$$
\begin{aligned}
g:[0,1] \times B V^{*} & \rightarrow B V \\
(\lambda, x) & \mapsto g(\lambda, x)
\end{aligned}
$$

tal que, para cada $s \in[0, T]$,

$$
g(\lambda, x)(s)=x(\lambda, 0)+\int_{0}^{T} f(\lambda, x(\lambda, t), t) d t+\int_{0}^{s} f(\lambda, x(\lambda, t), t) d t
$$

Notemos que o operador $\psi$ como definido em (3.48) pode ser escrito da seguinte forma:

$$
\psi(\lambda, x)(t)=i(\lambda, x)(t)-g(\lambda, x)(t), \quad t \in[0, T] .
$$

O resultado a seguir pode ser encontrado em [6] e nos diz que, sob certas condições existe ponto de bifurcação para a equação $\psi(\lambda, x)=0$. 
Teorema 3.27. Seja $\psi:[0,1] \times B V^{*} \rightarrow B V$, onde $\psi$ é o operador em (3.48). Suponha que $f:[0,1] \times \mathbb{R}^{n} \times[0, T] \rightarrow \mathbb{R}^{n}$ satisfaz (i), (ii) e (iii) e que exista $\lambda_{0} \in[0,1]$ tais que as seguintes condições são satisfeitas:

(i) $f(\lambda, 0, t)=0$, para todo $t \in[0, T], \lambda \in[0,1]$.

(ii) Para cada $\lambda \in[0,1] \backslash\left\{\lambda_{0}\right\}=J$, existe uma vizinhança $J_{\lambda} \times U_{\lambda}$ de $\left(\lambda_{0}, 0\right)$ em $J \times B V^{*}$ tal que 0 é o único zero de $g(\mu, \cdot)$ em $U_{\lambda}$, para todo $\mu \in J_{\lambda}$.

(iii) Existe uma função Lebesgue integrável $N:[0, T] \rightarrow \mathbb{R}$ tal que

$$
\left\|\int_{s}^{t} f\left(\lambda_{1}, x\left(\lambda_{1}, r\right), r\right) d r-\int_{s}^{t} f\left(\lambda_{2}, x\left(\lambda_{2}, r\right), r\right) d r\right\| \leq \int_{s}^{t}\left\|x\left(\lambda_{1}, r\right)-x\left(\lambda_{2}, r\right)\right\| N(r) d r
$$

para quaisquer $\lambda_{1}, \lambda_{2} \in[0,1]$ e $s, t \in[0, T]$.

Então $\left(\lambda_{0}, 0\right)$ será um ponto de bifurcação da equação $\psi(\lambda, x)=0$, se a indexação local, denotada por $\operatorname{ind}_{L S}[i-g(\lambda, \cdot), 0]$, satisfizer

$$
\operatorname{ind}_{L S}(i-g(0, \cdot), 0) \neq \operatorname{ind}_{L S}(i-g(1, \cdot), 0) .
$$





Apêndice

\section{A Integral de Kurzweil-Henstock}

Iniciaremos dando algumas definições básicas necessárias para o desenvolvimento da teoria de integração não absoluta de Kuzweil-Henstock.

\section{A.1 Calibres e partições}

Definição A.1. Dizemos que dois intervalos $[a, b],[c, d] \subset \mathbb{R}$ serão ditos quase disjuntos entre si se sua interseção for de, no máximo, um ponto.

Definição A.2. Seja $I=[a, b]$ um intervalo em $\mathbb{R}$, então uma divisão de I será uma coleção finita $\mathcal{D}=\left\{I_{i}: i=1, \ldots, n\right\}$ de intervalos $I_{i}=\left[t_{i-1}, t_{i}\right]$ quase disjuntos dois a dois tais que $I=I_{1} \cup I_{2} \cup \ldots \cup I_{n}$. 
Definição A.3. Uma divisão $\mathcal{D}$ será chamada de divisão marcada (abreviamos DM) se destacarmos, para cada subintervalo, um ponto $\xi_{i} \in\left[t_{i-1}, t_{i}\right]$ e em geral indicaremos $\mathcal{D}$ por $\left(\xi_{i}, t_{i}\right)$ e os pontos $\xi_{i}$ serão chamados de marcas da divisão.

Definição A.4. Definiremos por calibre de um intervalo $[a, b]$ qualquer a função $\delta:[a, b] \rightarrow$ $(0, \infty)$. Essa função $\delta$ calibra o intervalo em torno de $\xi_{i} \in\left[t_{i-1}, t_{i}\right]$, onde obtemos o intervalo $B(\xi ; \delta(\xi))=(\xi-\delta(\xi), t+\delta(\xi))$.

Definição A.5. Seja $\mathcal{D}$ uma $D M\left(\xi_{i}, t_{i}\right)$ de $[a, b]$. Dado um calibre $\delta$ de $[a, b]$ dizemos que uma $\operatorname{DM}\left(\xi_{i}, t_{i}\right)$ será $\delta$-fina, se para $i=1,2, \ldots, n$ teremos

$$
\left.\left[t_{i}, t_{i}\right] \subset\right] \xi_{i}-\delta\left(\xi_{i}\right), \xi_{i}+\delta\left(\xi_{i}\right)[
$$

Neste momento, destacamos que em uma $\operatorname{DM}\left(\xi_{i}, t_{i}\right)$ qualquer os pontos $t_{i}$ da divisão são são os elementos fundamentais e a marca $\xi_{i}$ é qualquer ponto em $\left[t_{i-1}, t_{i}\right]$, entretanto em uma DM $\delta$-fina os elementos fundamentais são as marcas $\xi_{i}$ e os intervalos devem se adaptar para satisfazer (A.1).

\section{A.2 Integral de Kurzweil-Henstock}

Com as definições anteriores estamos aptos a definir a integral de Kurzweil-Henstock.

Definição A.6. Dizemos que uma função $f:[a, b] \rightarrow \mathbb{R}$ será dita Kurzweil-Henstock integrável, ou simplesmente $K$-integrável e escreveremos $f \in K([a, b])$, se existir $I \in \mathbb{R}$ tal que para todo $\varepsilon>0$ existe um calibre $\delta:[a, b] \rightarrow(0, \infty)$ tal que para toda $D M \delta$-fina $\left(\xi_{i}, t_{i}\right)$ teremos

$$
\left|\sum_{i=1}^{n} f\left(\xi_{i}\right)\left(t_{i}-t_{i-1}\right)\right| \leq \varepsilon
$$

onde $n$ é o número de subintervalos de $[a, b]$ da divisão $\left(\xi_{i}, t_{i}\right)$, e escreveremos

$$
\int_{a}^{b} f(t)=I
$$


A definição da K-integral é muito semelhante a definição da integral de Riemann, entretanto veremos que a K-integral é bem mais geral. Primeiramente, podemos observar que a K-integral leva em consideração as peculiaridades da função a ser integrada. Assim, para diminuir a influência dos pontos $\xi$, cuja contribuição para a soma riemanniana é desproporcinal, diminuímos $\delta(\xi)$. Esta ideia nos permite ampliar muito a classe de funções integráveis.

Um segundo aspécto da K-integral que gostaríamos é que toda primitiva fosse uma integral. Isso não é verdade para a integral de Riemann. A seguir, denotaremos por $C([a, b])$ o conjunto das funções $f:[a, b] \rightarrow \mathbb{R}$ contínuas.

Teorema A.7 (Teorema Fundamental do Cálculo). Se $F \in C([a, b])$ for uma primitiva de $f:[a, b] \rightarrow \mathbb{R}$, isto é, se para todo $t \in[a, b]$ existir $F^{\prime}(t)=f(t)$, então $f \in K([a, b]) e$

$$
\int_{a}^{b} f(t)=F(a)-F(b) .
$$

Exemplo A.8. Consideremos a função de Dirichlet $f$ dada por

$$
f(x)= \begin{cases}1, & \text { se } x \in[0,1] \text { for racional } \\ 0, & \text { se } x \in[0,1] \text { for irracionl }\end{cases}
$$

Algumas rápidas considerações nos levam a ver que esta função é descontínua em todo ponto de $[0,1]$ e que $f$ não é Riemann integrável (R-integrável). Entretanto, mostraremos que $f$ é Kurzweil integrável e o valor de sua integral é 0 .

Se $\left\{r_{k}: k \in \mathbb{N}\right\}$ for uma enumeração dos números reais do intervalo $[0,1]$ e $\varepsilon>0$, um número arbitrário, definimos o calibre por

$$
\delta_{\varepsilon}(t):= \begin{cases}\varepsilon / 2^{k+1}, & t=r_{k}, \\ 1, & \text { se } t \text { for irracional } .\end{cases}
$$

Seja $\mathcal{D}$ uma $\operatorname{DM}\left(\xi_{i}, t_{i}\right) \delta$-fina de $[0,1]$. Se a marca $\xi_{i} \in I_{i}$ for irracional, então $f\left(\xi_{i}\right)=0$ e a contribuição do subintervalo para a soma de Riemann será 0. 
Se a marca $\xi_{i}$ for racional, então $f\left(\xi_{i}\right)=1$, mas o tamanho $l\left(I_{i}\right)=x_{i}-x_{i-1}$ é desprezível, já que $\mathcal{D}$ é $\delta_{\varepsilon}$-fina. Mais precisamente, se o k-ésimo número racional $r_{k}$ for a marca do intervalo $I_{i}$, então $I_{i} \subseteq\left(r_{k}-\delta_{\varepsilon}\left(\xi_{k}\right), r_{k}+\delta_{\varepsilon}\left(\xi_{k}\right)\right)$, de modo que $l\left(I_{i}\right) \leq 2 \delta_{\varepsilon}\left(r_{k}\right)=\varepsilon / 2^{k}$. Assim, se $r_{k}$ for a marca de dois subintervalos consecutivos em $\mathcal{D}$, então a soma dos tamanhos desses dois subintervalos será $\leq \varepsilon / 2^{k}$. Concluímos que o racional $r_{k}$ pode contribuir com, no máximo, $\varepsilon / 2^{k}$ para a soma de Riemann $S(f ; \mathcal{D})$. Como apenas marcas racionais fazem uma contribuição não nula para a soma de Riemann, temos

$$
|S(f ; \mathcal{D})| \leq \sum_{k=1}^{\infty} \frac{\varepsilon}{2^{k}} \leq \varepsilon .
$$

Como $\varepsilon>0$ é arbitrário, a função de Dirichlet é integrável e $\int_{0}^{1} f=0$.

O exemplo a seguir nos mostra que as funções Riemann integráveis são um subconjunto das funções Kurzweil integráveis.

Exemplo A.9. $R([a, b]) \subset K([a, b])$ e para $f \in R([a, b])$ temos que a $\int_{a}^{b} f(t)$ segundo Kurzweil, é igual a $\int_{a}^{b} f(t)$ segundo Riemann, onde $R([a, b])$ denota o espaço das funções de $[a, b]$ em $\mathbb{R}$ que são Riemann integráveis.

Podemos ver facilmente que isso vale pois, basta tomar o caibre constante igual a $2 \delta$ da definição de $R([a, b])$.

\section{A.3 Propriedades básicas da K-integral}

Antes de chegarmos no teorema da convergência monótona vamos enunciar algumas propriedades da K-integral.

Teorema A.10 (Lema de Causin). Dado um calibre $\delta:[a, b] \rightarrow(0, \infty)$, existe uma $D M\left(\xi_{i}, t_{i}\right)$ de $[a, b]$ que é $\delta$-fina, isto é, para todo $i=1,2, \ldots, n$ temos

$$
\left.\xi_{i} \in\left[t_{i-1}, t_{i}\right] \subset\right] \xi_{i}-\delta\left(\xi_{i}\right), \xi_{i}+\delta\left(\xi_{i}\right)[
$$


Muitas vezes precisamos que um certo ponto $c \in[a, b]$ seja uma marca de qualquer DM $\delta$-fina de $[a, b]$. Para isso, basta tomarmos $\delta$ tal que $c \notin\left(\xi_{i}-\delta\left(\xi_{i}\right), \xi_{i}+\delta\left(\xi_{i}\right)\right)$, se $\xi \neq c$ e é suficiente que tenhamos $\delta(\xi) \leq|\xi-c|$ se $\xi \neq c$. Nestas condições, dizemos que $\delta$ é c-especial. Assim, se $\delta$ é c-especial, então todo calibre $\delta^{\prime} \leq \delta$ também é c-especial e, se $\left(\xi_{i}, t_{i}\right)$ é uma DM $\delta$-fina $\operatorname{com} \xi_{n}=c \in(a, b)$, então temos $t_{k-1}<\xi_{k}=c<t_{k}$ ou $t_{k-1}<t_{k}=\xi_{k}=c=\xi_{k+1}<t_{k+1}$ (e analogamente se $c=a$ ou $c=b$ e fazendo a decompocição do intervalo $\left[t_{i-1}, t_{i}\right]$ no primeiro caso, podemos sempre supor que temos a segunda situação). Isso nos permite, em particular, decompor a soma riemanniana $\sum_{i=1}^{n} f\left(\xi_{i}\right)\left(t_{i}-t_{i-1}\right)$ numa soma correspondendo ao intevalo $[a, c]$ e outra correspondendo ao intervalo $[c, b]$

$$
\sum_{i=1}^{k} f\left(\xi_{i}\right)\left(t_{i}-t_{i-1}\right)+\sum_{j=k+1}^{n} f\left(\xi_{j}\right)\left(t_{j}-t_{j-1}\right)
$$

onde $k$ representa o número de divisões do intervalo $[a, c]$.

Teorema A.11 (Linearidade). $K([a, b])$ é um espaço vetorial e $f \in K([a, b]) \longmapsto \int_{a}^{b} f(t) \in \mathbb{R}$ é um funcional linear positivo (isto é, $f \geq 0 \Rightarrow \int_{a}^{b} f(t) \geq 0$ ).

Teorema A.12 (Aditividade). Sejam $c \in(a, b)$ e $f:[a, b] \rightarrow \mathbb{R}$ tais que $f \in K([a, c])$ e $f \in K([c, b])$. Então $f \in K([a, b]) e \int_{a}^{b} f(t)=\int_{a}^{c} f(t)+\int_{c}^{b} f(t)$.

Teorema A.13 (Unicidade). Se $f \in K([a, b])$, então o valor de sua integral é unicamente determinado.

Teorema A.14 (Critério de Cauchy). Seja $f:[a, b] \rightarrow \mathbb{R}$ tal que para todo $\varepsilon>0$, existe um calibre $\delta$ tal que, para quaisquer DM $\delta$-finas $\left(\xi_{i}^{\prime}, t_{i}^{\prime}\right)$ e $\left(\xi_{i}^{\prime \prime}, t_{i}^{\prime \prime}\right)$ de $[a, b]$ temos

$$
\left|\sum_{i=1}^{n} f\left(\xi_{i}^{\prime}\right)\left(t_{i}^{\prime}-t_{i-1}^{\prime}\right)-\sum_{j=1}^{m} f\left(\xi_{i}^{\prime \prime}\right)\left(t_{i}^{\prime \prime}-t_{i-1}^{\prime \prime}\right)\right|<\varepsilon
$$

onde n é o número e divisões de $[a, c]$ e $m$ é o número e divisões de $[c, b]$. 
Teorema A.15 (Integrabilidade nos subintervalos). Sejam $f \in K([a, b]) e[c, d] \subset[a, b]$. Então $f \in K([c, d])$.

Teorema A.16 (Lema de Saks-Henstock). Seja $f \in K([a, b])$ e seja $\delta$ um gauge de $[a, b]$ correspondente a $\varepsilon>0$ da definição de $\int_{a}^{b} f(t)$. Então para quaisquer

$$
a \leq c_{1} \leq n_{1} \leq d_{1} \leq c_{2} \leq n_{2} \leq d_{2} \leq \ldots \leq c_{m} \leq n_{m} \leq d_{m} \leq b
$$

$\left.\operatorname{com}\left[c_{j}, d_{j}\right] \subset\right] n_{j}-\delta\left(n_{j}, n_{j}+\delta\left(n_{j}\right)[\operatorname{para} j=1,2, \ldots, m\right.$, temos

$$
\left|\sum_{j=1}^{m}\left[f\left(n_{j}\right)\left(d_{j}-c_{j}\right)-\int_{c_{j}}^{d_{j}} f(t)\right]\right|<\varepsilon .
$$

Agora, vamos considerar

$$
\tilde{f}(t)=\int_{a}^{t} f(s), \text { para cada } a \leq t \leq b
$$

Corolário A.17. Seja $f \in K([a, b]) \operatorname{com} \tilde{f}=0$ e $g:[a, b] \rightarrow \mathbb{R}$ com $|g(t)| \leq|f(t)|$ para todo $t \in[a, b]$. Então $g \in K([a, b])$ e $\tilde{g}=0$.

Teorema A.18. Dado $f \in K([a, b])$ temos $\tilde{f} \in C([a, b])$, isto é, $\tilde{f}$ é contínua.

Teorema A.19. Seja $f:[a, b] \rightarrow \mathbb{R}$ tal que $f \in K([a, c])$ para todo $c \in[a, b[$ e tal que exista $\lim _{c \rightarrow b} \int_{a}^{b} f(t)=I \in \mathbb{R}$. Então $f \in K([a, b])$ e $\int_{a}^{b} f(t)=I$.

Teorema A.20 (Teorema da convergência monótona). Se $f_{n} \in K([a, b]), n \in \mathbb{N}$, são tais que para todo $t \in[a, b]$ temos $f_{1}(t) \leq f_{2}(t) \leq \ldots \leq f_{n}(t) \leq \ldots$ com $f_{n}(t) \rightarrow f(t)$ e $\sup _{n \in \mathbb{N}} \int_{a}^{b} f_{n}(t)=I<\infty$, então $f \in K([a, b])$ e $\int_{a}^{b} f(t)=I$. 


\section{Referências Bibliográficas}

[1] H. Amann, Ordinary differential equations. An introduction to nonlinear analysis.Gruyter Studies in Mathematics, 13. Walter de Gruyter Co., Berlin, 1990, xiv+458 pp.

[2] M. Braun, Equações Diferenciais e suas Aplicações, Campus, Rio de Janeiro, 1979, 378 pp.

[3] Z. G., Dennis, M. R. Cullen, Equações Diferenciais com Aplicações em Modelagem, Vol 1, São Paulo: Pioneira Thomson Learning, 2003, 491 pp.

[4] K. Deimling, Nonlinear Functional Analysis, Springer-Verlag, Berlin, 1985. xiv+450 pp.

[5] R. E. Gaines; J. L. Mawhin, Coincidence Degree and Nonlinear Differential Equations, Springer LNM vol. 568, Springer, Berlin, 1977.

[6] M. Federson, J. L. Mawhin, M. C. Mesquita, Periodic solutions of generalized ordinary differential equations and applications to bifurcation theory, 2013.

[7] J. Kurzweil, Generalized ordinary differential equations and continuous dependence on a parameter, Czechoslovak Math. J. 7(82) (1957), 418-449. 
[8] J. Kurzweil, Generalized ordinary differential equations, Czechoslovak Math. J., 8(83) (1958), 360-388.

[9] J. L. Mawhin, Leray-Schauder degree theory and mapping degree, J. Math. Anal. Appl. 402(1) (2013), 261-274.

[10] J. G. Mesquita; A. Slavík, Periodic averaging theorems for various types of equations, J. Math. Anal. Appl. 387(2) (2012), 862-877.

[11] I. P. Natanson, Theory of functions of a real variable. Vol. II. Translated from the Russian by Leo F. Boron Frederick Ungar Publishing Co., New York 1961265 pp.

[12] L. Perko, Differential equations and dynamical systems. Second edition. Texts in Applied Mathematics, 7. Springer-Verlag, New York, 1996. xiv+519 pp.

[13] Š. Schwabik, Generalized ordinary differential equations. Series in Real Analysis, 5. World Scientific Publishing Co., Inc., River Edge, NJ, 1992. x+382 pp.

[14] A. Slavík, Generalized differential equations: differentiability of solutions with respect to initial conditions and parameters, J. Math. Anal. Appl. 402(1) (2013), 261-274.

[15] M. Tvrdý, Differential and Integral Equations in the Space of Regulated Functions, Mem. Differential Equations Math. Phys., 25 (2002), 1-104. 